\title{
An In-Depth Study of Graph Partitioning Measures for Perceptual Organization
}

\author{
Padmanabhan Soundararajan, Student Member, IEEE, and Sudeep Sarkar, Member, IEEE
}

\begin{abstract}
In recent years, one of the effective engines for perceptual organization of low-level image features is based on the partitioning of a graph representation that captures Gestalt inspired local structures, such as similarity, proximity, continuity, parallelism, and perpendicularity, over the low-level image features. Mainly motivated by computational efficiency considerations, this graph partitioning process is usually implemented as a recursive bipartitioning process, where, at each step, the graph is broken into two parts based on a partitioning measure. We concentrate on three such measures, namely, the minimum [41], average [28], and normalized [32] cuts. The minimum cut partition seeks to minimize the total link weights cut. The average cut measure is proportional to the total link weight cut, normalized by the sizes of the partitions. The normalized cut measure is normalized by the product of the total connectivity (valencies) of the nodes in each partition. We provide theoretical and empirical insight into the nature of the three partitioning measures in terms of the underlying image statistics. In particular, we consider for what kinds of image statistics would optimizing a measure, irrespective of the particular algorithm used, result in correct partitioning. Are the quality of the groups significantly different for each cut measure? Are there classes of images for which grouping by partitioning does not work well? Another question of interest is if the recursive bipartitioning strategy can separate out groups corresponding to $K$ objects from each other. In the analysis, we draw from probability theory and the rich body of work on stochastic ordering of random variables. Our major conclusion is that optimization of none of the three measures is guaranteed to result in the correct partitioning of $K$ objects, in the strict stochastic order sense, for all image statistics. Qualitatively speaking, under very restrictive conditions, when the average interobject feature affinity is very weak when compared to the average intraobject feature affinity, the minimum cut measure is optimal. The average cut measure is optimal for graphs whose partition width is less than the mode of distribution of all possible partition widths. The normalized cut measure is optimal for a more restrictive subclass of graphs whose partition width is less than the mode of the partition width distributions and the strength of interobject links is six times less than the intraobject links. Rigorous empirical evaluation on 50 real images indicates that, in practice, the quality of the groups generated using minimum or average or normalized cuts are statistically equivalent for object recognition, i.e., the best, the mean, and the variation of the qualities are statistically equivalent. We also find that, for certain image classes, such as aerial and scenes with man-made objects, in man-made surroundings, the performance of grouping by partitioning is the worst, irrespective of the cut measure.
\end{abstract}

Index Terms-Perceptual organization, grouping, graph partitioning, stochastic orders, empirical evaluation.

\section{INTRODUCTION}

Derceptual organization refers to the process that groups 1 low-level image features based on emergent organization exhibited among them, without the use of specific object model knowledge. As a consequence of the generic nature of this process, it imparts flexibility to vision systems that are built upon it. This importance of the grouping process has been theoretically established by Clemens and Jacobs [8] in the context of indexing-based recognition and by Grimson [13] in the context of constrained search-based recognition, where the combinatorics reduce from an exponential to a low order polynomial if we use an intermediate grouping process.

Partitioning of a graph representation, defined over lowlevel image features based on Gestalt inspired relations, is an effective strategy for forming coherent perceptual groups in an image. The usual practice, mainly motivated

- The authors are with the Computer Science and Engineering Department, 4202 E. Fowler Ave., ENB 118, University of South Florida, Tampa, FL 33620. E-mail: \{psoundar, sarkar\}@csee.usf.edu.

Manuscript received 31 July 2001; revised 7 Aug. 2002; accepted 31 Jan. 2003. Recommended for acceptance by D. Jacobs and M. Lindenbaum.

For information on obtaining reprints of this article, please send e-mail to: tpami@computer.org, and reference IEEECS Log Number 118166. by efficiency considerations, is to approximate the general K-way partitioning solution by recursive bipartitioning, where, at each step, the graph is broken into two parts based on a partitioning measure. We concentrate on three such measures, namely, the minimum [41], average [28], and normalized [32] cuts. The minimum cut partition seeks to minimize the total link weight cut. The average cut measure is proportional to the total link weight cut, normalized by the sizes of the partitions. The normalized cut measure is normalized by the product of the total connectivity (valency) of the nodes in each partition.

The questions we ask in this work are: For what kinds of image statistics would optimizing each of the three measures, irrespective of the particular algorithm used, result in correct grouping of the low-level features? Another question of interest is if the recursive bipartitioning strategy can separate out groups corresponding to $K$ objects from each other. Do the nature of the cut measures really matter? Are the quality of the groups significantly different for each cut measure? Are there classes of images on which grouping by partitioning does not work well?

The contributions of this paper are two-fold: First, we analytically relate the nature of each partitioning measure 
to the underlying image statistics. This lets us quantify under what conditions minimizing each measure would give us the correct partitions. Our major conclusion is that optimization of none of the three measures is guaranteed to result in the correct partitioning into $K$ objects, in the strict stochastic order sense, for all image statistics. Qualitatively speaking, under very restrictive conditions, when the average interobject feature affinity is very weak when compared to the average intraobject feature affinity, the minimum cut measure is optimal. The average cut measure is optimal for graphs whose partition width is less than the mode of distribution of all possible partition widths. The normalized cut measure is optimal for a more restrictive subclass of graphs whose partition width is less than the mode of the partition width distributions and the strength of interobject links is six times less than the intraobject links. Second, we empirically evaluate the groups produced by graph partitioning, based on the three measures, viz. min-cut, average cut, and normalized cut, given the task of grouping extended edge segments. Our findings in this regard suggest that the quality of the groups with each of these measures is statistically equivalent, as far as object recognition is concerned. We also examine whether the performance of the grouping-by-partitioning strategy depends on the image class.

The exposition of this paper is organized as follows: In the next section, we review the relevant work, mainly the graph-based approaches to perceptual organization and other related performance comparison work. In Section 3, we present our theoretical analyses and work out the relationships between the cut measures and the underlying image statistics. In Section 4, we present an analysis of the actual performance of the cut measures on real images. We conclude by discussing the implications of our findings in Section 5 .

\section{Relevant Prior Work}

One of the most common approaches to grouping is based on graph representations that capture the structure amongst low-level primitives such as image pixels, edge pixels, straight lines, arcs, and region patches. The links of the graph, which are typically weighted, capture the association or affinity between the primitives. There are two different classes of approaches for forming groups from this graph representation. First is the class of techniques that search for special graph structures such as cycles [22], [16], [17], [26], [18], cliques [26], spanning trees [42], or shortest paths [26], [6]. The second class of techniques seeks to find clusters of graph nodes based on some global coherence criterion [27], [39], [21], [28], [32], [41], [12], [37], [10]. In particular, we look at techniques that seek to form these node clusters by partitioning the graph.

$\mathrm{Wu}$ and Leahy [41] proposed the concept of using minimum cuts for image segmentation. They constructed a graph whose nodes represent pixels and links indicate affinities. A sparse graph was created by using a suitable threshold for the link weights. Clusters were formed by recursively finding the minimum cuts of this graph using an algorithm based on the Ford-Fulkerson theorem.

Gdalyahu et al. [12] approach the graph partitioning problem by stochastic clustering. They partition the graph into $k$ parts by inducing a probability distribution over each cut that decreases monotonically with its capacity.

Shi and Malik [32] suggested the novel normalized cut measure for grouping edge pixels. The normalized cut measure is the ratio of the edges cut to the product of connectivity (valency) of the nodes in each partition. Perona and Freeman [21] considered an asymmetric version of the Shi and Malik normalized cut measure. The Perona and Freeman cut is the ratio of the edges cut to the total edges cut in the foreground objects.

Sarkar and Soundararajan [28] also use a graph partitionbased framework, but for grouping constant curvature edge segment primitives. Their partition metric can be shown to be equivalent to minimizing the edges cut, normalized by the product of the sizes of each partition and, hence, can be termed as the average cut. Both the normalized and the average cuts can be well approximated by a solution constructed out of the graph spectra. A graph spectrum is the set of eigenvalues and eigenvectors of the matrix representation (e.g., adjacency, Laplacian, normalized Laplacian, etc.) of the graph.

The works relevant to probabilistic analysis of the grouping algorithm are the analyses performed by Amir and Lindenbaum [1] and Berengolts and Lindenbaum [2]. Their analysis is based on a binomial distributed cue. The number of background points falsely added to the group is used to quantify the grouping quality. They provide an upper bound on the number of false additions to the foreground. Their analysis is done on complete graphs as well as on locally dense graphs ( $k$-connected). Recently, Berengolts and Lindenbaum [2] analyzed the connected components algorithm and used a probabilistic model to derive expressions for addition errors and the group fragmentation rate, taking into account interfering or nonindependent cues.

Three studies that considered comparison of different graph clustering methods are those of Weiss [38], who studied similarities of graph spectral methods for segmentation, Williams and Thornber [40], who considered clustering methods based on the affinity matrix, and Matula [19], who considered clustering methods based on the proximity matrix. Weiss compared four different spectral algorithms, namely, Perona and Freeman [21], Shi and Malik [32], Scott and Longuet-Higgins [29], and Costeira and Kanade [9], and proposed one of his own combinations for segmentation. In his analysis, Weiss considered an image with two clusters with different but constant within cluster and between cluster affinities. He extended his analysis to the case when the variation of the within and between cluster dissimilarities are small and to the case when between cluster affinities are zero. He found that 
Scott and Longuet-Higgins performs well for constant blocks (clusters of the same size) and Shi-Malik performs well for nonconstant blocks.

Williams and Thornber consider the association-based saliency measures of Shashua and Ullman [30], Herault and Hourad [15], Sarkar and Boyer [27], Guy and Medioni [14], and Williams and Jacobs [39]. They also propose a new saliency measure that defines salience of an edge to be the relative number of closed random walks that visit that edge. They first compare these saliency measures on simple test patterns consisting of (30,20, and 10 uniformly spaced) edges from a circle in a background of 100 edges of random position and orientation. Performance was quantified based on the computed saliencies of the object (circle) edges. In the second part of the study, they used edge-detected $32 \times 32$, images of nine different fruits and vegetables in front of a uniform background. To simulate realistic noisy backgrounds, they used nine Canny edge detected textured images as mask patterns. The test patterns were then constructed by ANDing the vegetable silhouettes into the center $32 \times 32$ regions of the $64 \times 64$ sized edge detected textures. For their test setup, they use a total of 405 patterns with different signal-to-noise ratios and discuss just the false positive rate compared to the noisy edges. However, the strategy for choosing the parameters for each of the saliency measures is not clear.

Matula [19] used the connectivity feature of the graph to induce subgraphs of the proximity graph. He derives three measures for clustering methods, namely, $k$-bond, $k$-component, and $k$-block. These metrics are based on a cohesiveness function, which was defined for all nodes and edges of the graph, as the maximal edge-connectivity of any subgraph containing $k$ elements. He also mentions briefly about applying these measures on random proximity graphs.

Our study complements and extends the previous studies in grouping in that we 1) consider $K$-objects in the scene, instead of just two, for the theoretical analysis, 2) relate the distribution of the cut measures to the underlying image statistics, and 3) undertake a rigorous and extensive empirical evaluation. The empirical evaluation is conducted on real images, using a rigorous automated parameter selection process, and thorough statistical analyses.

\section{Theoretical Analyses}

There is a rich body of prior work on the analysis of graph cuts. Most works consider the classic problem of minimum cut or the problem of graph bisection, which has origins in VLSI. The graph bisection problem involves computing the minimum cut with constraints on the sizes of the partitions such as the oft-used equal sized partitions requirement. The minimum cut problem is a well-studied one that has also been extended to partitioning into $k$-parts [25]. While the minimum cut problem without any constraint on partition size has polynomial complexity, the problem of graph bisection with equal sized partitions is NP-complete. In fact, Wagner and Wagner [36] showed that the problem of graph bisection with unequal partition sized constraints, with minimum partition size that is $\mathcal{O}\left(\alpha N^{\epsilon}\right)$, is also NP-hard. Because of this computational challenge there is interest in the design of approximate algorithms or optimal algorithms for restricted graph classes. One of the earlier works toward this end is by Bui et al. [5], who described a polynomial time algorithm that computes the minimum bisection, optimally for $d$-regular random graphs and bisection width of $b$. Later, Boppana [3] showed that graph bisection could be computed, for almost all graphs, by maximizing the largest eigenvalue of the transformation of adjacency matrix of the graph. The analysis is based on a random graph model that involves $n$ vertices with $m$ edges, with a bisection width of b. More recently, $\mathrm{Yu}$ and Cheng [35] showed that the Boppana bisection could also be computed efficiently using semidefinite programming. For $k$-regular graphs, Saab and Rao [23] showed that the greedy strategy for finding the graph bisection could find approximate solutions that are close to the optimal one.

Our analysis is different in many respects from traditional analyses of graph cuts. One fundamental difference is that we are concerned with analyzing the cut measures themselves and not concerned with the optimality of particular algorithmic strategies used to solve the problem. For example, we would like to know if recursively minimizing the average cut would result in correct groups. Would it result in correct groups for all image statistics? We are not interested, at least in this section, in designing algorithms that find the optimal average cut of a given graph instance. Finding an optimal solution to, say, the average cut problem, is not useful if minimizing it does not result in groups that segregate objects from each other. The second difference is that, in addition to the minimum cut measure, we consider the average and normalized cut measures, which are relatively new. The graph bisection and even the generalized $k$-section version, that have been studied quite a bit is not appropriate in the vision context since we do not a priori know the number of features from each object. The third new aspect is the modeling of the partition space (f-space), that we use; it is appropriate only in the context of the object recognition problem. This restricted context helps in managing the exponential size of the partition space. Another direction in which we push the state of art, at least in the context of graph-based grouping methods, is in that we consider weighted graphs.

We provide theoretical insight into the nature of the three partitioning measures in terms of the underlying image statistics. In particular, we consider for what kinds of image statistics would optimizing a measure, irrespective of the particular algorithm used, result in correct partitioning. Another question of interest is if the recursive bipartitioning strategy can separate out groups corresponding to $K$ objects from each other. For the analysis, we draw from probability theory and the rich body of work on stochastic ordering of 


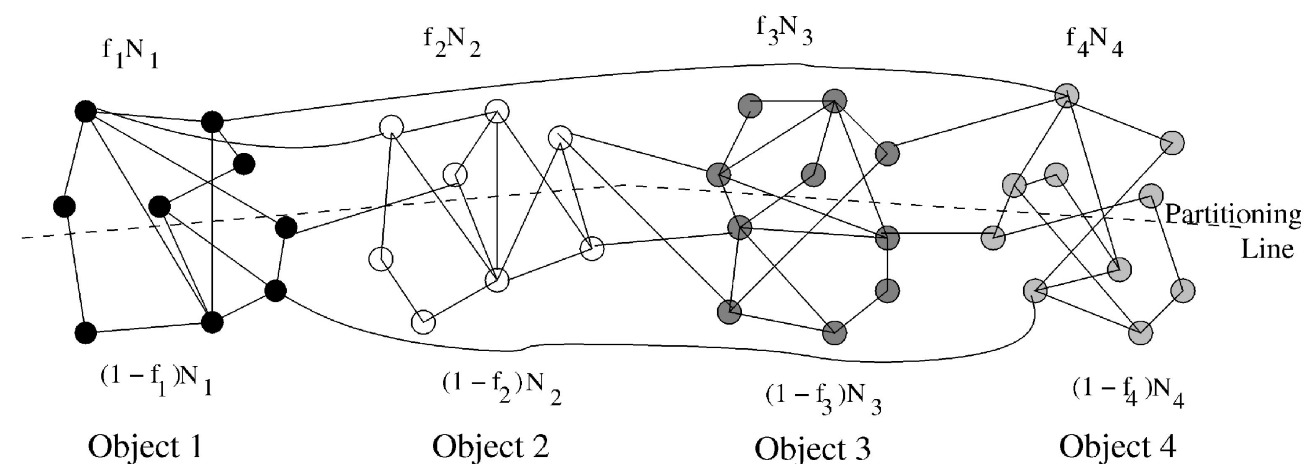

Fig. 1. Partitioning of a scene structure graph over features from multiple objects. (See text for explanation of notations.)

random variables [31]. Our major conclusion is that none of the three measures is guaranteed to result in the correct partitioning of $K$ objects, in the strict stochastic order sense, for all image statistics. Qualitatively speaking, under very restrictive conditions when the average interobject feature affinity is very weak when compared to the average intraobject feature affinity, the minimum cut measure is optimal. The normalized cut measure is partially optimal, i.e., optimal over a restricted range of cut values, when interobject mean feature affinity is somewhat weak than the mean intraobject affinity. And, the average cut measure is also partially optimal, but with the least restrictive requirement that the mean interobject affinity be just less than the mean intraobject affinity.

In our analysis, we assume that we have a weighted scene structure graph, with positive valued weights, which is true for most grouping strategies. Fig. 1 depicts the notations that we use in this section and formally define below.

\section{Definition 1. Let}

1. the number of objects be denoted by $K$ and the object themselves be denoted by $O_{1}, \cdots, O_{K}$,

2. the number of features of the $i$ th object be denoted by $N_{i}$, and

3. the weights of a link, $X_{l m}^{i}$, between the lth and $m$ th features (or nodes) from the same ith object be a Gamma random variable with

$$
P\left(X_{l m}^{i}=x\right)=\operatorname{Gamma}(\Omega)=\frac{1}{\Gamma(\Omega)} x^{\Omega-1} e^{-x},
$$

where $\Gamma(x)$ is the standard gamma function. Recall that Gamma random variables take on values between 0 and $\infty$. The mean and the variance are both $\Omega$ and the mode is $\Omega-1$. The parameter $\Omega$ is also known as the shape parameter.

4. the weights of a link, $Y_{l m}^{i j}$, between the lth feature from the $i$ th object and the $m$ th feature from the jth object be a Gamma random variable with

$$
P\left(Y_{l m}^{i j}=y\right)=\operatorname{Gamma}(\omega)=\frac{1}{\Gamma(\omega)} y^{\omega-1} e^{-y} .
$$

We can assume that the strength of the association between interobject features will be lower than that between intraobject features.

Assumption 1. Both the mean and the mode of the weight distribution for links between features from different objects are lower than that for links between features from the same object, i.e., $\omega<\Omega$. This, based on the theory presented in next section, would imply that $Y_{l m}^{i j}<_{l t} X_{l m}^{i}$, i.e., $Y_{l m}^{i j}$ is stochastically less than $X_{l m}^{i}$.

In the 50 real images that we have experimented with, the estimated $\frac{\Omega}{\omega}$ ratio is around 7 . Fig. 2 shows the fit of the gamma model to distribution of link weights, both between inter and intraobject features.

We also use the oft assumed property that

Assumption 2. The link weights are independent random variables.

We need notations to characterize a bipartition of a multiobject association graph. Instead of representing each possible partition individually, which is combinatorially explosive, we represent the possible bipartition classes as follows:

Definition 2. A bipartition result in two partitions $\left(S_{1}\right.$ and $\left.S_{2}\right)$ such that $f_{i} N_{i}$ features from the $i$ th object are in one partition $\left(S_{1}\right)$ and the rest of the $\left(1-f_{i}\right) N_{i}$ features are in the other partition $\left(S_{2}\right)$. A class of equivalent bipartitions is characterized by the column vector: $\mathbf{f}=\left(f_{1}, \cdots, f_{K}\right)^{T}$.

Note that $f_{i}$ s are discrete numbers that range from 0 to 1 in increments of $\frac{1}{N_{i}}$. For recursive bipartitioning to eventually result in the correct $K$-way cut, $f_{i}$ should be 0 or 1 , excluding the case when all $f_{i}$ s are 0 or all $f_{i}$ s are 1 . In the context of object recognition, all partitions in a bipartition classes are considered to be equivalent in computational terms of how they would impact object recognition. The underlying assumption in this context is that all features from the same object are equally important. This assumption is not new and has been made by others when analyzing object recognition systems, such as by Clemens and Jacobs [8] in the context of indexing-based recognition and by Grimson [13] for constrained searchbased recognition. 


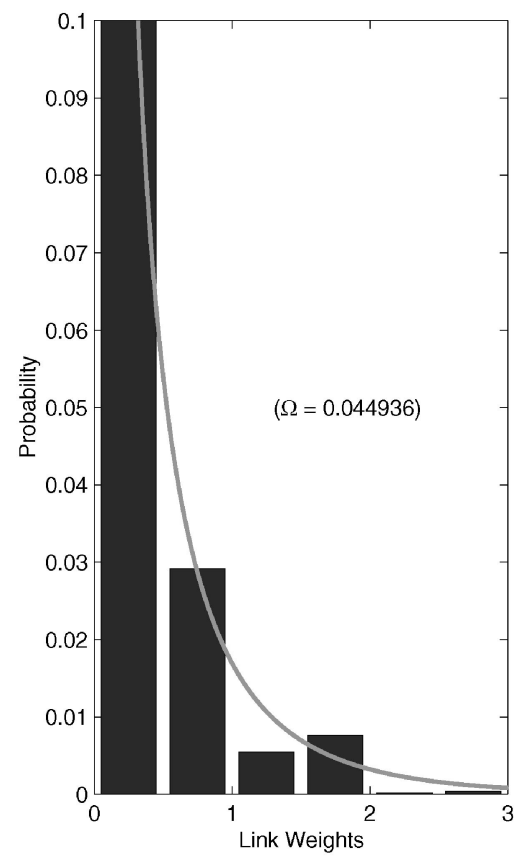

(a)

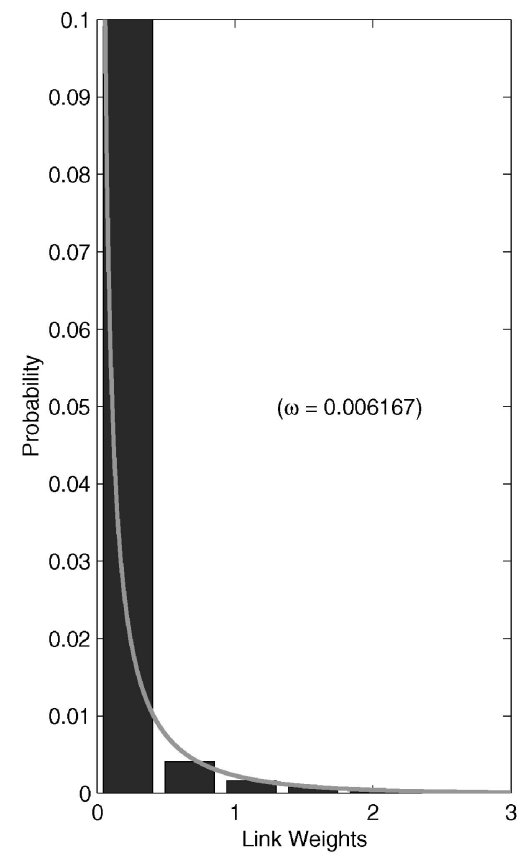

(b)

Fig. 2. Empirical fit of the gamma probability density function to link weight distribution: (a) for links between same object features and (b) for links between features from different objects.

It is trivial to show that

Lemma 1. The partition class $\left(f_{1}, \cdots, f_{K}\right)$ is equivalent to the partition class $\left(1-f_{1}, \cdots, 1-f_{K}\right)$.

Definition 3. Let $\mathbf{0}$ and $\mathbf{1}$ denote vectors whose components are all 0 and 1 , respectively.

Definition 4. Let $\Delta$ denote the set of vectors $\delta$, each of whose components, $\delta_{i}$ is either 0 or 1 , excluding the vectors $\mathbf{0}$ and $\mathbf{1}$. The dimension of $\delta$ is the same as that of $\mathbf{f}$.

Definition 5. Let $\Phi$ denote the set of vectors $\phi$, whose ith components is either 0 or $\frac{1}{N_{i}}$, excluding the vector $\mathbf{0 .}$

Definition 6. Let $\Psi$ denote the set of vectors $\psi$, whose $i$ th components is either 1 or $1-\frac{1}{N_{i}}$, excluding the vector 1 .

The corner points on the boundary of the domain of possible partitioning classes is given by the set $\{\Delta \cup \Phi \cup \Psi\}$. That the elements of $\Delta$ will be boundary corner points is obvious. The elements in the sets $\Psi$ and $\Phi$ arise because we exclude $\mathbf{f}=\mathbf{0}$ and $\mathbf{f}=\mathbf{1}$, which do not represent a "partition." We also make use of the fact that the possible values for $f_{i}$ are $\frac{k}{N_{i}}$ for $k=0,1, \cdots, N_{i}$. Partitions represented by the elements in $\Psi$ and $\Phi$ are undesirable partitions that separate just one feature of some object(s) from the others. The elements of $\Delta$ represent the set of desired partition classes, where none of the individual objects are partitioned.

Using the above notations and assumptions, we next establish the probability models for cut weight, association within each partition, and the number of features in each partition. Note that the sizes of each partition class $\mathbf{f}$ will not appear in the analysis since we are interested in algorithm independent characteristics. The use of partition class sizes would be relevant when analyzing a particular partitioning strategy that makes choices about different partition classes in a selected manner.

Lemma 2. The total link weights cut, $\Sigma_{c}(\mathbf{f})$, for partitions in the class $\mathbf{f}$ is a Gamma random variable, $\operatorname{Gamma}(\kappa(\mathbf{f}))$, where $\kappa(\mathbf{f})=\mathbf{f}^{T} \mathbf{P}(\mathbf{1}-\mathbf{f})$ and $\mathbf{P}$ is a $K \times K$ matrix with

$$
\mathbf{P}(i, j)= \begin{cases}\Omega N_{i}^{2} & \text { for } i=j \\ \omega N_{i} N_{j} & \text { for } i \neq j .\end{cases}
$$

Proof. The cut links are of two kinds, interobject and intraobject links. The total weight of the cut interobject links is a sum of individual Gamma variables specified by $\sum_{i \neq j} \sum_{l} \sum_{m} Y_{l m}^{i j}$. The number of the intraobject links is a random variable given by $\sum_{i} \sum_{l} \sum_{m} X_{l m}^{i}$. These sums are also Gamma distributed, which follows from the property that, if $X_{1}$ is $\operatorname{Gamma}\left(\kappa_{1}\right)$ and $X_{2}$ is $\operatorname{Gamma}\left(\kappa_{2}\right)$, then $X_{1}+X_{2}$ is $\operatorname{Gamma}\left(\kappa_{1}+\kappa_{2}\right)$.

Thus, $\sum_{i} \sum_{l} \sum_{m} X_{l m}^{i}$ is $\operatorname{Gamma}\left(\Omega \sum_{i} f_{i}\left(1-f_{i}\right) N_{i}^{2}\right)$ and $\sum_{i \neq j} \sum_{l} \sum_{m} Y_{l m}^{i j}$ is $\operatorname{Gamma}\left(\omega \sum_{i \neq j} f_{i}\left(1-f_{j}\right) N_{i} N_{j}\right)$. The sum of these two sums will again be a Gamma random variable, whose shape parameter can be compactly expressed in the above specified matrix notation by using $\mathbf{P}$, which is a $K X K$ matrix as specified in the lemma.

Lemma 3. The number of features in each partition can be expressed as

$$
\begin{aligned}
& \operatorname{Size}_{S_{1}}(\mathbf{f})=\mathbf{f}^{T} \mathbf{N} \\
& \operatorname{Size}_{S_{2}}(\mathbf{f})=(\mathbf{1}-\mathbf{f})^{T} \mathbf{N},
\end{aligned}
$$

where $\mathbf{N}$ is a column vector whose ith entry is $N_{i}$.

Proof. This follows trivially from $\operatorname{Size}_{S_{1}}(\mathbf{f})=\sum_{i} f_{i} N_{i}$ and Size $_{S_{2}}(\mathbf{f})=\sum_{i}\left(1-f_{i}\right) N_{i}$. 


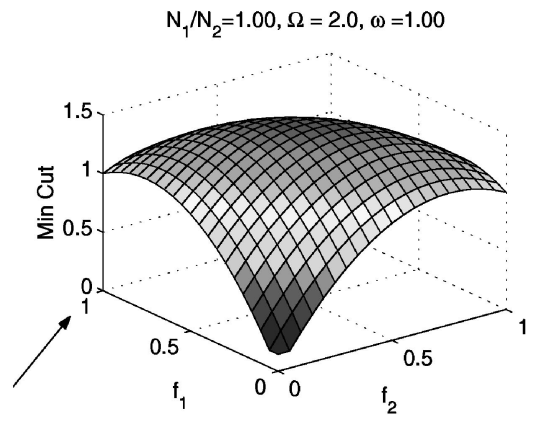

(a)

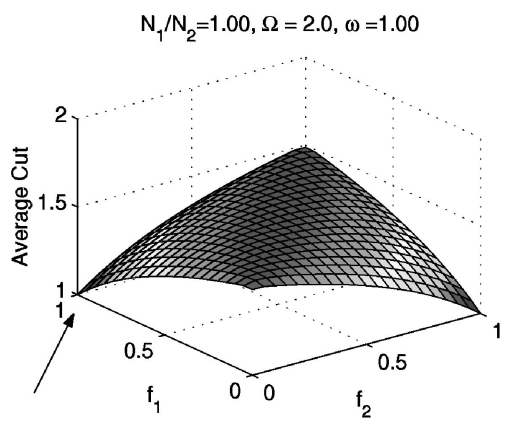

(b)

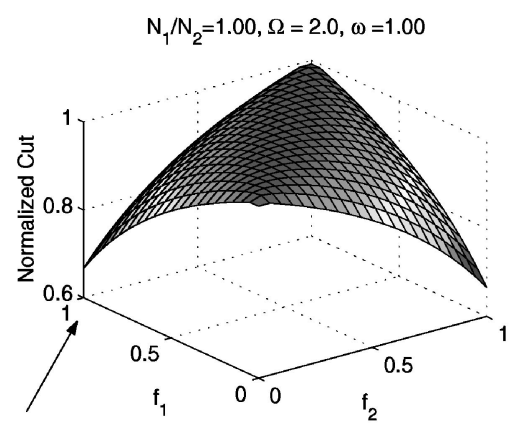

(c)

Fig. 3. The expected values of the three measures, (a) minimum cut, (b) average cut, and (c) normalized cut measures plotted as a function of $f_{1}$ and $f_{2}$ for a scene with similar sized objects and with the strength of connection within objects being just twice the strength between objects, i.e., $N_{1}=N_{2}, \Omega=2$, and $\omega=1$.

Lemma 4. The sum of the link weights, $\Sigma_{1}$ and $\Sigma_{2}$, in the two partitions, $S_{1}$ and $S_{2}$, respectively, from the partition class, $\mathbf{f}$, are Gamma distributed random variable given by

$\Sigma_{1}(\mathbf{f})$ is Gamma $\left(\mathbf{f}^{T} \mathbf{P}^{\prime} \mathbf{f}-\mathbf{f}^{T} \mathbf{Q}\right)$

$\Sigma_{2}(\mathbf{f}) \quad$ is $\operatorname{Gamma}\left((\mathbf{1}-\mathbf{f})^{T} \mathbf{P}^{\prime}(\mathbf{1}-\mathbf{f})-(\mathbf{1}-\mathbf{f})^{T} \mathbf{q}\right)$,

where $\mathbf{P}^{\prime}$ is a $K X K$ matrix defined as,

$$
\mathbf{P}^{\prime}(i, j)= \begin{cases}0.5 \Omega N_{i}^{2} & \text { for } i=j \\ \omega N_{i} N_{j} & \text { for } i \neq j\end{cases}
$$

and $\mathbf{q}$ is a column vector of size $K$ with entries $\mathbf{q}(i)=0.5 \Omega N_{i}$, where $i \in 1, \ldots, K$.

Proof. We use the fact that the sum of gamma random variables is also a gamma random variable whose shape parameter is the sum of the shape parameters of the constituent random variables. Within each part, separately counting the inter and intraobject links we have

$$
\begin{aligned}
\Sigma_{1}(\mathbf{f}) \text { is Gamma }( & \sum_{i=1}^{K} 0.5 \Omega\left(f_{i}^{2} N_{i}^{2}-f_{i} N_{i}\right)+ \\
& \left.\sum_{i \neq j} \omega f_{i} f_{j} N_{i} N_{j}\right)
\end{aligned}
$$

$\Sigma_{2}(\mathbf{f}) \quad$ is Gamma $\left(\sum_{i=1}^{K} 0.5 \Omega\left(\left(1-f_{i}\right)^{2} N_{i}^{2}-\left(1-f_{i}\right) N_{i}\right)+\right.$

$$
\left.\sum_{i \neq j} \omega\left(1-f_{i}\right)\left(1-f_{j}\right) N_{i} N_{j}\right) .
$$

Using the matrix $\mathbf{P}^{\prime}$, we can compactly express the shape parameter as specified in this lemma.

\subsection{Comparing Random Variables: Stochastic Orders}

In our analysis, we will derive expressions for the probability density functions describing the distributions of the cut measures as a function of the partition classes, denoted by $\Sigma(\mathbf{f})$. We will have to compare these random variables to establish optimality. Specifically, we will compare the random variables representing cut values for partition classes in $\Delta$ with those that are not $\Delta$. We would like to know if $\Sigma(\mathbf{f} \in \Delta)<\min _{\mathbf{f} \notin \Delta} \Sigma(\mathbf{f})$. The simplest way is to compare the mean values of two random variables, which has been our earlier strategy [33]. As an example of this type of comparison, Figs. 3 and 4 show the nature of the variation of the expected values of the three cut measures as a function of all possible partitions, $\left(f_{1}, f_{2}\right)$, of an image with two object, for two different image statistics. In each figure, the desired partition, namely, $\left(f_{1}=1, f_{2}=0\right)$, is represented by the corner of the space, indicated by an arrow. (In the following sections, we derive exact expressions for these mean values. We preview these means just for illustration of the nature of the variation.) Notice that, both the expected value of average cut and the normalized cut measures seem to be well formed with a minimum at the right partition, while the expected minimum cut does not always have a minimum at the 


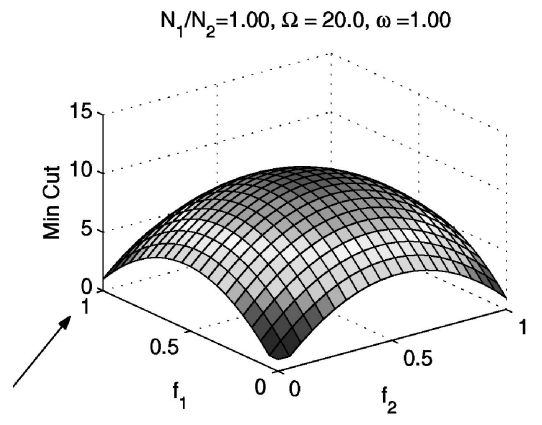

(a)

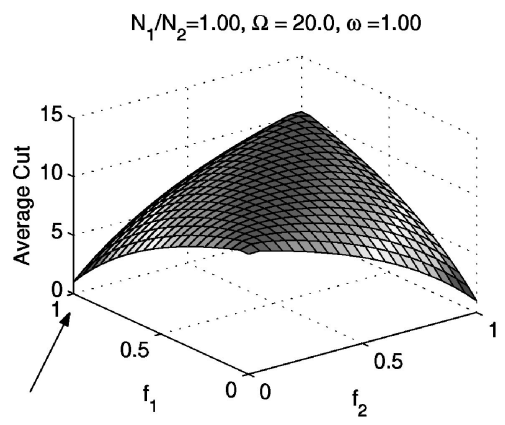

(b)

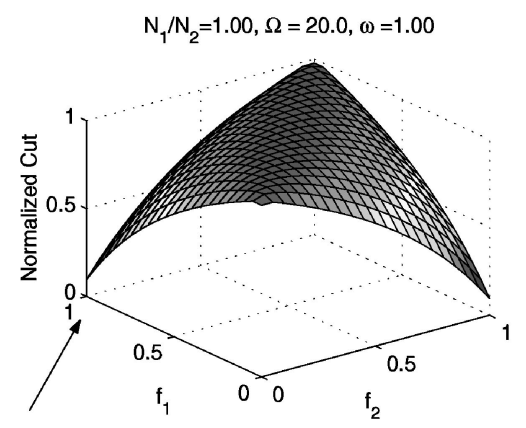

(c)

Fig. 4. The expected values of the three measures, (a) minimum cut, (b) average cut, and (c) normalized cut measures plotted as a function of $f_{1}$ and $f_{2}$ for a scene with similar sized objects and with the strength of connection within objects being 20 times the strength between objects, i.e., $N_{1}=N_{2}$, $\Omega=20$, and $\omega=1$.

correct partition. Thus, the expected value of the average and normalized cut measure for partitions that do not split object features is lower than those partitions that do split feature from the same object into two partitions. These visual observations regarding the mean values of the cuts have been analytically proven in [33].

This comparison of means is somewhat informative, but does not establish strong results. Hence, we turn to the body of work in stochastic orders that establishes definitions for comparing random variables. In this section, we present some of the key concepts and results that we will use in our subsequent analyses. For an extensive exposition of stochastic orders, we refer the reader to the excellent book by Shaked and Shanthikumar [31].

An extremely strong way to define ordering of random variables $X$ and $Y$ is to insist that every realization of $X$ be less than $Y$, i.e., $\operatorname{Prob}(X<Y)=1$. However, this is an overly restrictive definition, applicable only for very few real world situations. There are other ways of defining stochastic ordering that are less strong, but widely applicable. Interestingly, some of these orderings can be related to the strong ordering sense through proper transformations. Among the many ways of defining stochastic ordering between random variables, we pick two that are of particular interest to us.

Definition 7. A random variable $X$ is stochastically less than $Y$, $X \leq_{s t} Y$, if $P(X<t) \geq P(Y<t)$ for every $t$.
In other words, one random variable is less than another one in the stochastic sense when it is more likely for $X$ than for $Y$ to have values less than any given number. Intuitively, this sense of stochastic order is appealing but is sometimes mathematically hard to establish, so, we usually consider the following sense.

Definition 8. A random variable $X$ is less than $Y$ in the likelihood order sense, $X \leq_{l r} Y$, if $\frac{P(X=t)}{P(Y=t)}$ is a decreasing in $t$ over the union of the supports of $X$ and $Y$.

In other words, lower values are more likely for $X$ and for $Y$. Although this sense of stochastic order seems to be unintuitive, it turns out that it is easier to establish this mathematically than the stochastic sense and is the stronger of the two senses. The following properties are of particular interest to us.

1. If $X \leq_{l r} Y$, then $X \leq_{s t} Y$. In other words, the likelihood ratio sense is a stronger sense of stochastic order.

2. $X \leq_{s t} Y$ if and only if there exists two random variables $\hat{X}$ and $\hat{Y}$, defined on the same probability space such that $\hat{X}={ }_{s t} X$ and $\hat{Y}_{s t}=Y$ and $P(\hat{X} \leq Y)=1$. Notice the connection to the strongest sense of stochastic order.

3. If $X \leq_{l r} Y$, then $f(X) \leq_{l r} f(Y)$, where $f$ is an increasing function.

4. If $X \leq_{l r} Y$, then $E[f(X)] \leq_{l r} E[f(Y)]$ where $f$ is an increasing function. Thus, statistical properties such as the mean and moments of $X$ would be less than those for $Y$. 
5. Let $\left(X_{i}, Y_{i}\right), i=1,2$ be independent pairs of random variables such that $X_{i} \leq_{l r} Y_{i}$ for $i=1,2$. If $X_{i}, Y_{i}$ are log-concave densities, then $X_{1}+X_{2}<_{l r} Y_{1}+Y_{2}$.

6. Let $X$ be a random variable independent of $Y_{i}, i=1, \cdots, N$. If $X \leq_{s t} Y_{i}$ for $i=1, \cdots, N$ then $X \leq_{s t} \min _{i} Y_{i}$. This property is important to establish optimality in the presence of the minimizing operation involved in the graph partitioning operation.

7. Let $X$ and $Y$ be gamma random variables with parameters $\left(\kappa_{1}, b_{1}\right)$ and $\left(\kappa_{2}, b_{2}\right)$, respectively. If for every $t, \Lambda\left(k_{1}, b_{1}\right) \leq \Lambda\left(k_{2}, b_{2}\right)$, where $\Lambda(k, b)=k-\frac{t}{b}$, then $X \leq_{l r} Y$. This is easily established by taking a derivative of the ratio of the two pdfs and requiring that it be less than zero. In the analysis, the normalizing constants drop out and we are left with just the terms involving the random variable values. We will refer to $\Lambda$ as the likelihood ratio ordering characteristic function. If the inequality between the $\Lambda$ s is true only for a range of $t$, then the corresponding random variables are ordered only over that range. Note that for the ordering to be valid over all $t, k_{1}$ and $b_{1}$ should be less than $k_{2}$ and $b_{2}$, respectively.

8. Let $X$ and $Y$ are beta random variables ${ }^{1}$ with parameters $\left(a_{1}, b_{1}\right)$ and $\left(a_{2}, b_{2}\right)$, respectively. The likelihood ratio ordering characteristic function for two beta variables is given by $\Lambda(a, b)=a+t(b-a)$. If for every $t, \Lambda\left(a_{1}, b_{1}\right) \leq \Lambda\left(a_{2}, b_{2}\right)$, then $X \leq_{l r} Y$. Note that, for the ordering to be valid over all $t, a_{1}$ and $b_{1}$ should be less than $a_{2}$ and $b_{2}$, respectively.

\subsection{Minimum Cut}

A minimum cut-based graph bipartition will try to minimize the total weight of cut links $\left(\Sigma_{c}\right)$, whose distribution as a function of the partition class are specified in Lemma 2. Ideally, we would like each recursive bipartitioning not to split features from a single object into two groups. In other words, the minimum value of the cut should happen for partitions of the type $\mathbf{f} \in \Delta$ or $f_{i}=\delta_{i}$. In particular, we are interested in the event $\Sigma_{c}(\delta)<\min _{\mathbf{f} \notin \Delta} \Sigma_{c}(\mathbf{f})$. Using Property 6 from the previous section, it would suffice to establish the conditions under which $\Sigma_{c}(\delta)<\Sigma_{c}(\mathbf{f} \notin \Delta)$. To answer this question, we first establish a few lemmas about the behavior of the shape parameter governing the distribution of $\Sigma_{c}$, i.e., $\kappa(\mathbf{f})=\mathbf{f}^{T} \mathbf{P}(\mathbf{1}-\mathbf{f})$.

Lemma 5. The function, $\kappa(\mathbf{f})=\mathbf{f}^{T} \mathbf{P}(\mathbf{1}-\mathbf{f})$, is a concave function in the space of partition classes $\mathbf{f}$.

Proof. Let $f_{1}$ and $f_{2}$ be two partition classes. A linear combination of these two vectors is given by $\alpha \mathbf{f}_{1}+(1-\alpha) \mathbf{f}_{2}$, where $\alpha \in(0,1)$. For a concave function, the following relation must be true.

$\left(\alpha \mathbf{f}_{\mathbf{1}}+(1-\alpha) \mathbf{f}_{\mathbf{2}}\right)^{T} \mathbf{P}\left(\mathbf{1}-\alpha \mathbf{f}_{\mathbf{1}}-(1-\alpha) \mathbf{f}_{\mathbf{2}}\right)>$

$\alpha \mathbf{f}_{\mathbf{1}}{ }^{T} \mathbf{P}\left(\mathbf{1}-\mathbf{f}_{\mathbf{1}}\right)+(1-\alpha) \mathbf{f}_{\mathbf{2}}{ }^{T} \mathbf{P}\left(\mathbf{1}-\mathbf{f}_{\mathbf{2}}\right)\left(\mathbf{f}_{\mathbf{2}}-\mathbf{f}_{\mathbf{1}}\right)^{T} \mathbf{P}\left(\mathbf{f}_{\mathbf{2}}-\mathbf{f}_{\mathbf{1}}\right)>0$.

1. If $X$ is a beta distribution, then the probability density function is given by $p(x)=\frac{1}{B(a, b)} x^{a-1}(1-u)^{b-1}$ for $0<x<1$, where $B(a, b)$ is the beta function.
In deriving the above, we have used the facts that 1) $\mathbf{f}_{\mathbf{1}}{ }^{T} \mathbf{P} \mathbf{f}_{\mathbf{2}}=\mathbf{f}_{\mathbf{2}}{ }^{T} \mathbf{P} \mathbf{f}_{\mathbf{1}}$, since $\mathbf{P}$ is a symmetric matrix and 2) $\alpha(1-\alpha)>0$. The above-required condition can be rearranged into

$$
\mathbf{F}^{T} \mathbf{p F}>0
$$

where $\mathbf{F}_{\mathbf{1}}(i)=N_{i}\left(\mathbf{f}_{\mathbf{1}}(i)-\mathbf{f}_{\mathbf{2}}(i)\right)$, and

$$
\mathbf{p}(i, j)= \begin{cases}\Omega & \text { for } i=j \\ \omega & \text { for } i \neq j .\end{cases}
$$

This transformed condition would be true if $\mathbf{p}$ is a positive definite matrix, which it is. The matrix $\mathbf{p}$ can be expressed as the sum of a diagonal matrix, with positive diagonal entries $\Omega-\omega$, and a constant matrix, all of whose entries are $\omega$. Since the diagonal matrix is positive definite and the constant matrix is positive semidefinite, their sum is positive definite.

Lemma 6. The partition classes, $\mathbf{f}$, that minimize the function $\kappa(\mathbf{f})=\mathbf{f}^{T} \mathbf{P}(\mathbf{1}-\mathbf{f})$ would also maximize $\left|\operatorname{Size}_{S_{1}}(\mathbf{f})-\operatorname{Size}_{S_{2}}(\mathbf{f})\right|$.

Proof. We prove this by contradiction. Let us assume that $\mathbf{f}$ minimizes $\kappa(\mathbf{f})$, but the corresponding $\mid \operatorname{Size}_{S_{1}}(\mathbf{f})-$ Size $_{S_{2}}(\mathbf{f}) \mid$ is not the maximum possible. We show that we can derive an $\mathbf{f}^{\prime}$ with a lower value of $\kappa\left(\mathbf{f}^{\prime}\right)$, but with larger difference in sizes than this assumed minimum partition.

Let us consider the $k$ th component of $\mathbf{f}$, representing the partition of the features from the $k$ th object. The function $\kappa(\mathbf{f})$ can be expressed as the sum of two types of terms: the terms that include $f_{k}$ and those that do not. We denote the aggregate of the terms that do not include $k$ th object by $K$. We can then express $\kappa(\mathbf{f})$ as:

$$
\begin{aligned}
\kappa(\mathbf{f})=K & +p_{k k} f_{k}\left(1-f_{k}\right) N_{k}^{2}+\left(\sum_{j \neq k} p\left(1-f_{j}\right) N_{j}\right) f_{k} N_{k} \\
& +\left(\sum_{j \neq k} p f_{j} N_{j}\right)\left(1-f_{k}\right) N_{k} \\
=K & +p_{k k} f_{k}\left(1-f_{k}\right) N_{k}^{2}+\sum_{j \neq k} p f_{j} N_{j} N_{k} \\
& +\left(\sum_{j \neq k} p\left(\left(1-f_{j}\right) N_{j}-f_{j} N_{j}\right)\right) f_{k} N_{k} \\
=K & +p_{k k} f_{k}\left(1-f_{k}\right) N_{k}^{2}+\sum_{j \neq k} p f_{j} N_{j} N_{k} \\
& +p\left(N_{+}-N_{-}\right) f_{k} N_{k},
\end{aligned}
$$

where we used $N_{+}$and $N_{-}$to denote $\sum_{j \neq k}\left(1-f_{j}\right) N_{j}$ and $\sum_{j \neq k} f_{j} N_{j}$, respectively. Note that $N_{+}$and $N_{-}$represent the size of the two partitions excluding object $k$.

If $\left(N_{+}>N_{-}\right)$, then choosing $f_{k}=0$ will give us a lower value for $\kappa(\mathbf{f})$, which also results in a partitioning vector whose $\left(\operatorname{Size}_{S_{2}}(\mathbf{f})-\operatorname{Size}_{S_{1}}(\mathbf{f})\right)$ is larger than our starting vector. If $\left(N_{+}<N_{-}\right)$, then choosing $f_{k}=1$ will give us a lower value for $\kappa(\mathbf{f})$. In this case, too, the resulting partitioning vector would have $\left|\operatorname{Size}_{S_{2}}(\mathbf{f})-\operatorname{Size}_{S_{1}}(\mathbf{f})\right|$ that is larger than for our starting vector. 
As a consequence of the above two lemmas, we have

Corollary 1. The possible candidates for the minimum value of $\kappa(\mathbf{f})$ are those partitions in $\Delta, \Phi$, and $\Psi$ that have only one component that is different from all the others, i.e., the set $\left\{\left\{\phi \mid \sum_{i} \phi_{i}=\frac{1}{N_{i}}\right\} \cup\left\{\psi \mid \sum_{i} \psi_{i}=1-\frac{1}{N_{i}}\right\} \cup\left\{\delta \mid \sum_{i} \delta_{i}=1\right\}\right\}$.

This follows from the facts that 1$)$ the $\kappa(\mathbf{f})$ is a concave function, hence, the minimum will be achieved by partitions represented by the boundary of the domain and 2) these candidates represent the most disparate sized partitions on the boundary.

Theorem 1. The recursive partitioning strategy based on the minimization of the cut values will result in correct groups, in the likelihood ratio-based stochastic order sense, if $\omega<\frac{\Omega}{N-N_{1}}$, where $N_{1}=\min \left\{N_{1}, \cdots, N_{K}\right\}$.

Proof. The assertion would be true if $\Sigma_{c}(\mathbf{f} \in \Delta)$, the cut value for correct partition classes, is less than $\Sigma_{c}(\mathbf{f} \notin \Delta)$. Since these random variables are gamma distributed (Theorem 2), $\Sigma_{c}(\mathbf{f} \in \Delta) \leq_{l r} \Sigma_{c}(\mathbf{f} \notin \Delta)$, if $\kappa(\mathbf{f} \in \Delta) \leq \kappa(\mathbf{f} \notin \Delta)$, where the $\kappa$ s are the shape parameters of the corresponding densities. From Corollary 1, we know that the lowest value of $\kappa$ will be for partition classes in $\Psi, \Phi$, or $\Delta$.

$$
\kappa(\mathbf{f} \in \Phi)=(\Omega-\omega) N_{i}+(\omega N-\Omega)
$$

and

$$
\kappa(\mathbf{f} \in \Delta)=\omega N_{i}\left(N-N_{i}\right) .
$$

The minimum value of the above two $\kappa$ s will be for the case when $N_{i}$ is the minimum possible.

The required condition, i.e., $\kappa(\mathbf{f} \in \Phi)>\kappa(\mathbf{f} \in \Delta)$ can be transformed as follows:

$$
\left(\Omega-\omega\left(N-N_{i}\right)\right)\left(N_{i}-1\right)>0 .
$$

The above will be always true if $\omega<\frac{\Omega}{N-N_{1}}$, where $N_{1}=\min \left\{N_{1}, \cdots, N_{K}\right\}$. Since the vectors in $\Psi$ represent the same partitions as the one in $\Phi$, we do not need any other condition.

\subsection{Average Cut}

We first establish that the average cut values are general gamma distributed variables, whose parameters are functions of the partition classes. This, we follow by an enunciation of the conditions under which minimizing this measure will lead to correct partitioning in the stochastic sense. We find that for graphs with partition widths, i.e. the minimum average cut value, is less than $\omega N$, minimizing the average cut makes sense.

Lemma 7 . The average cut value $\Sigma_{\text {avg }}(\mathbf{f})$ for partition in the partition class $\mathbf{f}$ is a gamma random variable distributed according to

$$
\operatorname{Gamma}\left(\mathbf{f}^{T} \mathbf{P}(\mathbf{1}-\mathbf{f}), \frac{N}{\mathbf{f}^{T} \mathbf{N} \mathbf{N}^{T}(\mathbf{1}-\mathbf{f})}\right) .
$$

Proof. The average cut measure of a partition is the total cut link weight normalized by the product of the sizes of the two partitions.

$$
\Sigma_{a v g}(\mathbf{f})=N \frac{\Sigma_{c}(\mathbf{f})}{\operatorname{Size}_{S_{1}}(\mathbf{f}) \operatorname{Size}_{S_{2}}(\mathbf{f})} .
$$

Thus, the average cut measure for partitions in $\mathbf{f}$ is a gamma random variable that is scaled by product of the partition size. Recall that, if $X$ is $\operatorname{Gamma}(\kappa)$, then $b X$ is general $\operatorname{Gamma}(\kappa, b)$ distribution. ${ }^{2}$ The final expression follow from this observation and the expressions in Theorem 2 and Lemma 3.

Lemma 8. The mean value of the average-cut cost, $\mu_{\text {avg }}(\mathbf{f})$, attains its minimum at $\mathbf{f} \in \Delta$ and this minimum is a constant $\omega N$.

Proof. The matrix $\mathbf{P}$ can be expressed as sum of a diagonal and nondiagonal matrix: $\mathbf{P}=\mathbf{P}_{1}+\mathbf{P}_{2}$, where

$$
\mathbf{P}_{\mathbf{1}}(i, j)= \begin{cases}(\Omega-\omega) N_{i}^{2} & \text { for } i=j \\ 0 & \text { for } i \neq j\end{cases}
$$

and

$$
\mathbf{P}_{2}(i, j)=\omega N_{i} N_{j}
$$

It is easy to see that $\mathbf{P}_{\mathbf{2}}=\omega \mathbf{N} \mathbf{N}^{T}$. Thus, the expression for the expected average cut is given by

$$
\mu_{a v g}(\mathbf{f})=N \frac{\sum_{i}(\Omega-\omega) f_{i}\left(1-f_{i}\right) N_{i}^{2}}{\sum_{i} \sum_{j} f_{i}\left(1-f_{j}\right) N_{i} N_{j}}+\omega N .
$$

Using Assumption 1, we can easily see that the first term on the right hand side is always positive and will attain a minimum value of 0 whenever all $f_{i}$ s are 0 or 1 , in other words $f \in \Delta$. The denominator of the first term is never 0 for valid partitions $\mathbf{f} \in \Delta$. Thus, the minimum value of $\omega N$ is attained by partition vectors in $\Delta$.

Theorem 2. The average cut measure $\Sigma_{\text {avg }}(\mathbf{f})$ will result in a minimum, in the stochastic order sense over a restricted range $(0, \omega N)$, for partitions in $\Delta$. This restricted range always includes the mode and the mean of the optimal cut values.

Proof. We would like to establish that $\Sigma_{\text {avg }}(\mathbf{f} \in \Delta) \leq_{s t}$ $\min _{\mathbf{f} \notin \Delta} \Sigma_{\text {avg }}(\mathbf{f})$. Based on Properties 1 and 6 from Section 3.1, we just need to show that $\Sigma_{\text {avg }}(\mathbf{f} \in \Delta) \leq_{l r}$ $\Sigma_{a v g}(\mathbf{f} \notin \Delta)$. For this to be true, the likelihood ratio ordering characteristic function, $\Lambda$, should attain a minimum for $\mathbf{f} \in \Delta$. Using Property 7 from Section 3.1 and the expression derived earlier in this section, the function $\Lambda$ for average cut values can be expressed as

2. The general Gamma distribution given by $\frac{1}{\Gamma(\kappa) b}\left(\frac{x}{b}\right)^{\kappa-1} e^{-\frac{x}{b}}$ for $x>0$, where $b$ is the scaling factor. 


$$
\begin{aligned}
\Lambda(\kappa(\mathbf{f}), b(\mathbf{f})) & =\kappa(\mathbf{f})-\frac{t}{b(\mathbf{f})} \\
& =\mathbf{f}^{T} \mathbf{P}(\mathbf{1}-\mathbf{f})-\frac{t}{N} \mathbf{f}^{T} \mathbf{N N}^{T}(\mathbf{1}-\mathbf{f}) \\
& =\mathbf{f}^{T}\left(\mathbf{P}-t \frac{\mathbf{N N}}{N}\right)(\mathbf{1}-\mathbf{f}) .
\end{aligned}
$$

Using a derivation similar to that in the proof for Lemma 5 , we can show that the function $\Lambda(\kappa(\mathbf{f}), b(\mathbf{f}))$ is concave if $\mathbf{p}-\frac{t}{N} \mathbf{1 1 ^ { T }}$ is positive definite, where $\mathbf{p}$ is as defined in Lemma 5. This condition results in the requirement that $t<\omega N$.

Using this concavity property, we can say that $\Lambda(\mathbf{f} \in$ $\{\Delta, \Psi, \Phi\}) \leq \Lambda(\mathbf{f} \notin\{\Delta, \Psi, \Phi\})$ for $t<\omega N$. We can also infer that $\Sigma_{a v g}(\mathbf{f} \in\{\Psi, \Phi\}) \not_{l r} \Sigma_{a v g}(\mathbf{f} \in \Delta)$ using the fact that the minimum of mean of the $\Sigma_{\text {avg }}$ is achieved for $\mathbf{f} \in \Delta$ (Lemma 8). Thus, we have $\Sigma_{\text {avg }}(\mathbf{f} \in \Delta) \leq_{l r} \Sigma_{\text {avg }}(\mathbf{f} \notin \Delta)$ but only for $t<\omega N$. Note that $\omega N$ is also the mean value of cut value for optimal partitions, $\Sigma_{\text {avg }}(\mathbf{f} \in \Delta$ ) (from Lemma 8); since for gamma random variables the mode is less than or equal to the mean, the assertion of the theorem follows.

This theorem suggests that minimizing the average cut is appropriate for graphs whose partition width, or the minimum average cut value for the graph, is less than $\omega N$. It can be easily established that $\operatorname{Pr}(t<\omega N)>0.5$ using the fact the median of a gamma random variable is always less than the mean value, which in this case is $\omega N$.

\subsection{Normalized Cut}

We show that the normalized cut is a sum of two betadistributed random variables, whose parameters are functions of the partition classes. Using this, we derive the condition that, if $\Omega>3 \omega$ and the partition width, i.e., the minimum value of the normalized cut value, is less than 0.5 , minimizing the normalized cut measure makes sense.

Lemma 9. The normalized cut value $\Sigma_{n r m}(\mathbf{f})$ for partitions in the partition class $\mathbf{f}$ is a sum of two beta random variables, $\Sigma_{n r m}^{1}(\mathbf{f})+\Sigma_{n r m}^{2}(\mathbf{f})$. The random variable $\Sigma_{n r m}^{1}(\mathbf{f})$ is Beta $(\kappa(\mathbf{f}), a(\mathbf{f}))$ and $\Sigma_{n r m}^{2}(\mathbf{f})$ is Beta $(\kappa(\mathbf{f}), b(\mathbf{f}))$, where the parameters are

$$
\begin{gathered}
\kappa(\mathbf{f})=\mathbf{f}^{T} \mathbf{P}(\mathbf{1}-\mathbf{f}) \\
a(\mathbf{f})=\mathbf{f}^{T} \mathbf{P}^{\prime} \mathbf{f}-\mathbf{f}^{T} \mathbf{q} \\
b(\mathbf{f})=(1-\mathbf{f})^{T} \mathbf{P}^{\prime}(1-\mathbf{f})-(1-\mathbf{f})^{T} \mathbf{q}
\end{gathered}
$$

Proof. The normalized cut measure of a partition is the total cut link weight normalized by the sum of the inverses of the connectivities of the partitions. The connectivity of each partition is the sum of the valencies of the nodes in that partition, which can be expressed as the sum of the valencies within each partition and the cut value. Using the notations from Lemmas 2 and 3, the normalized cut random variable can be expressed as

$$
\Sigma_{n r m}(\mathbf{f})=\frac{\Sigma_{c}(\mathbf{f})}{\Sigma_{c}(\mathbf{f})+\Sigma_{1}(\mathbf{f})}+\frac{\Sigma_{c}(\mathbf{f})}{\Sigma_{c}(\mathbf{f})+\Sigma_{2}(\mathbf{f})} .
$$

We have also established in Lemmas 2 and 3 that $\Sigma_{c}, \Sigma_{1}$, and $\Sigma_{2}$ are all gamma random variables. The claims of this lemma follow from this and the basic property that, if $X_{1}$ and $X_{2}$ are independent gamma distributed random variables with scale parameter $b$ and shape parameters $\kappa_{1}$ and $\kappa_{2}$, respectively, then $\frac{X_{1}}{X_{1}+X_{2}}$ is a beta distributed random variable with parameters $\kappa_{1}$ and $\kappa_{2}$. $\square$

Corollary 2. The mean and mode of the normalized cut value $\Sigma_{n r m}(\mathbf{f})$ for partition in the partition class $\mathbf{f}$ are given by

$$
\begin{gathered}
\mu_{n r m}(\mathbf{f})=\frac{\kappa(\mathbf{f})}{\kappa(\mathbf{f})+a(\mathbf{f})}+\frac{\kappa(\mathbf{f})}{\kappa(\mathbf{f})+b(\mathbf{f})} \\
\operatorname{Mode}_{n r m}(\mathbf{f})=\frac{\kappa(\mathbf{f})-1}{\kappa(\mathbf{f})+a(\mathbf{f})-2}+\frac{\kappa(\mathbf{f})-1}{\kappa(\mathbf{f})+b(\mathbf{f})-2} .
\end{gathered}
$$

Note that, for $\mathbf{f} \in \Delta$, when we expect $\kappa<a$ or $b$, the mode will be less than the mean value.

Theorem 3. The normalized cut measure $\Sigma_{n r m}(\mathbf{f})$ will result in a minimum, in the stochastic order sense over a restricted range $(0,0.5)$, for partitions in $\Delta$. This restricted range will include the mode and the mean of the optimal cut values if the $\omega<\frac{\Omega}{6} \frac{N_{1}-1}{N-N_{1}}$, where $N_{1}=\min \left\{N_{1}, \cdots, N_{K}\right\}$.

Proof. Property 5 from Section 3.1 establishes the conditions for sum of two beta random variables to be stochastically less than sum of two other beta variables. Thus, we need to show that $\Sigma_{n r m}^{i}(\mathbf{f} \in \Delta)<_{s t} \min _{\mathbf{f} \notin \Delta} \Sigma_{n r m}^{i}(\mathbf{f})$, for $i=1,2$. We sketch the proof for $\Sigma^{1}$ and the proof for $\Sigma^{2}$ is along similar lines.

The required condition is that the likelihood ratio ordering characteristic function, $\Lambda$, should attain a minimum for $\mathbf{f} \in \Delta$. Using Property 8 from Section 3.1 and the expression derived earlier in this section, the function $\Lambda$ for normalized cut values can be expressed as

$$
\begin{aligned}
\Lambda(\kappa(\mathbf{f}), a(\mathbf{f})) & =\kappa(\mathbf{f})+t(a(\mathbf{f})-\kappa(\mathbf{f})) \\
& =\mathbf{f}^{T} \mathbf{P}(\mathbf{1}-\mathbf{f})+t\left(\mathbf{f}^{T} \mathbf{P}^{\prime} \mathbf{f}-\mathbf{f}^{T} \mathbf{q}-\mathbf{f}^{T} \mathbf{P}^{\prime}(\mathbf{1}-\mathbf{f})\right) \\
& =\mathbf{f}^{T} \mathbf{P}^{\prime \prime}(\mathbf{1}-\mathbf{f})+t \mathbf{f}^{T}(\mathbf{P} \mathbf{1}-\mathbf{q}),
\end{aligned}
$$

where,

$$
\mathbf{P}^{\prime \prime}(i, j)= \begin{cases}(1-t) \Omega N_{i}^{2} & \text { for } i=j \\ (1-2 t) \omega N_{i} N_{j} & \text { for } i \neq j .\end{cases}
$$

For the required condition, this function $\Lambda(\kappa(\mathbf{f}), a(\mathbf{f}))$ should be concave, which is true only for $t<0.5$.

Using this concavity property, we can say that $\Lambda(\mathbf{f} \in$ $\{\Delta, \Psi, \Phi\}) \leq_{s t} \Lambda(\mathbf{f} \notin\{\Delta, \Psi, \Phi\})$ for $t<0.5$. We can also infer that $\Sigma_{n r m}^{1}(\mathbf{f} \in\{\Psi, \Phi\}) \not_{l r} \Sigma_{n r m}^{1}(\mathbf{f} \in \Delta)$ by comparing the corresponding means. Thus, we have $\Sigma_{n r m}^{1}(\mathbf{f} \in \Delta) \leq_{l r} \Sigma_{n r m}^{1}(\mathbf{f} \notin \Delta)$, but only for $t<0.5$. We can derive the same condition for $\Sigma_{n r m}^{2}$. 
For this range, $t<0.5$, to include the mean and the mode normalized cut values of correct partitions, $(\mathbf{f} \in \Delta)$, we can show using the expression for the mean that $3 \kappa(\mathbf{f})<\min (a(\mathbf{f}), b(\mathbf{f}))$. If we assume that the smallest object size is $N_{1}$, then for $\mathbf{f} \in \Delta$, $\min (a(\mathbf{f}), b(\mathbf{f}))=0.5 \Omega N_{1}\left(N_{1}-1\right)$ and the corresponding $\kappa(\mathbf{f})=\omega(\mathbf{f}) N_{1}\left(N-N_{1}\right)$. From these, we can derive the required condition to be $\omega<\frac{\Omega}{6} \frac{N_{1}-1}{N-N_{1}}$; the strength (as measured by the mean values) of the connections inside the smallest object should be more than six times larger than that between the objects.

Thus, we have results similar to, but somewhat more restrictive conditions than that for the average cut measure. Minimizing the normalized cut is appropriate for graphs whose partition width, or the minimum normalized cut value for the graph, is less than 0.5 and the within-object connections are at least six times stronger than the betweenobject connections. Since the mean and the mode of the cut values for $\mathbf{f} \in \Delta$ are included in the range $(0,0.5)$, we conjecture that the median is also included in this range, at least for $\mathbf{f} \in \Delta$ and, so, $\operatorname{Pr}(t<0.5)>0.5$.

\section{Empirical Evaluation}

The prediction from theory is that the optimization of each of the partition measures should result in groups of varying quality. While the minimization of the average cut and the normalized cut measures should, at least more than 50 percent of the time, be able to correctly group features from multiple objects, the optimization of the minimum cut measure will not be able to do so under all image statistics conditions. However, in practice, slight degradation in group quality does not really matter. The empirical question we ask is whether the quality of the groups produced by recursive minimization of the three measures are sufficiently different to significantly impact object recognition. To answer this question, we empirically evaluate the groups produced by graph partitioning based on the min-cut, average cut, and normalized cut, given the task of grouping constant curvature edge segments for object recognition by constrained searchbased techniques. We augment the min-cut strategy with additional logic to prevent splintering of small groups of features, which it is prone to do. We simply do not partition if size of one of the resulting partitions is smaller than a user specified minimum cluster size.

The hypothesis of this empirical study is that the quality of the groups of constant curvature segments obtained by minimizing the three partition measures, i.e., min-cut, average cut, and normalized cut, are not sufficiently different to impact object recognition in a statistically significant manner.

The road map for this section is as follows: First, we discuss the strategy for the construction and quantification of the scene structure graph, which we will partition to form the groups. Second, we outline how we implement each of the partitioning techniques. Third, we discuss the performance measure used. Fourth, we discuss the strategy for parameter selection; then, we present extensive statistical analyses.

\subsection{The Scene Structure Graph}

For this study, we consider the grouping of extended image edge features, namely, constant curvature segmentsstraight line segments and circular arcs. Other possible choices of the low-level primitives might be pixels or region patches, which are not considered here. The grouping framework is essentially the same as that described in [28]. We present just the sketch of the essential aspects.

The Gestalt inspired relationships of parallelism, perpendicularity ( $\mathrm{T}$ and L-junctions), proximity, continuity, and common region form the basis for the formulation of the link weights between any two nodes representing the constant curvature segments. The links are quantified based on the following attributes computed between any two edge segments.

1. The maximum and the minimum distance of the end points of the smaller segments to the larger segment, normalized by the length of the larger segment.

2. The overlap between the two segments.

3. The minimum distance between the end points of the two segments, normalized by the length of the larger segment.

4. The difference in slope.

5. In addition to the above geometric attributes, we compute two photometric attributes, $r_{\text {mag }}$ and $r_{\text {width }}$, that are based on the response of the second derivative of the smoothed image function near the edge. At the edge, this response is of course zero, however, away from the edge the response peaks to a maximum on one side of the edge and to a minimum on the other side. These peaks capture the behavior of the image function on either side of the edge. For each edge segment we compute the averages along the segment of the magnitudes $\left(r^{+}, r^{-}\right)$and the distances $\left(w^{+}, w^{-}\right)$of these extremum points from the edge location. We quantify the photometric attributes between two edge segments $e_{i}$ and $e_{j}$ by

$$
r_{\text {mag }}=\max \left(\frac{\left|r_{i}^{+}-r_{j}^{+}\right|}{0.5\left(r_{i}^{+}+r_{j}^{+}\right)}, \frac{\left|r_{i}^{-} r_{j}^{-}\right|}{0.5\left(r_{i}^{-}+r_{j}^{-}\right)}\right)
$$

and

$$
w_{\text {width }}=\max \left(\frac{\left|w_{i}^{+}-w_{j}^{+}\right|}{0.5\left(w_{i}^{+}+w_{j}^{+}\right)}, \frac{\left|w_{i}^{-} w_{j}^{-}\right|}{0.5\left(w_{i}^{-}+w_{j}^{-}\right)}\right) .
$$

Based on these photometric and geometric attributes, we classify and quantify the relationship between each pair of edge segment as being parallel, T-junction, L-junction, continuous, or "none-of-the-above." This is achieved using the maximum a posteriori probability (MAP) strategy based on the conditional probabilities of inferring the relationships based on the computed attributes. The a posteriori 
probabilities are computed using Bayesian networks, which efficiently and succinctly encode the relational priors and the a priori conditional probabilities. These priors and conditional probabilities are expressed in parametric forms whose parameters form the parameters of the grouping system. We also use Bayesian networks to quantify the proximity and the common region factors between two edge segments in a MAP fashion. The sum of these three maximum a posteriori probabilities forms the weights of the scene structure graph that would be partitioned.

\subsection{Graph Partitioning Implementations}

The average and the normalized cut measures-based partitioning can be well approximated using graph spectral partitioning strategies. This techniqueinvolves the calculation of the second eigenvector of the Laplacian matrix representation of the scene structure graph. For the average cut, we use the unnormalized Laplacian matrix and for the normalized cut, we use the normalized version. We refer the reader to [32], [28] for more details about the supporting theory.

We use the LEDA-4.0 implementation of the min-cut algorithm. The implementation is based on the algorithm proposed by [34]. The algorithm uses the concept of Prim's minimum spanning tree algorithm. The algorithm starts with a node in the graph and keeps adding the most loosely connected node to this original node. In doing so, it builds up subsets of nodes, which eventually leads to the full set of nodes in the graph. During this process, the cost of adding each loosely connected node to the subset is maintained, which is used to identify the minimum cost partition(s).

\subsection{Performance Measure}

We evaluate the quality of the final groups in terms of the expected performance of a constrained search-based object recognition strategy starting from the groups. In [4], Borra and Sarkar proposed performance measures that do not require building a full vision system, but instead use manually specified ground truth. Let 1) $N_{G_{i}}$ denote the number of features in the detected group $\left.\left(G_{i}\right), 2\right) N_{O_{j}}$ denote the number of model features (ground truth) in $O_{j}$, and 3) $N_{G_{i} \cap O_{j}}$ denote the number of group features that lie on the model. We can construct two performance measures that capture the expected time and quality of matching group $G_{i}$ with model $O_{j}$ as follows.

We start from the expression for complexity of constrained search-based object recognition using imperfect groups. Assuming that all features are equally important, Grimson [13] showed that the expected search, $W_{\text {term }}$, is essentially polynomial if we terminate when the number of matched features equal some predetermined threshold, $t$. The exact expression is given by:

$$
\begin{aligned}
& N_{O_{j}} N_{G_{i}} \frac{N_{G_{i}}}{N_{G_{i} \cap O_{j}}} \leq \\
& W_{\text {term }} \leq t N_{O_{j}} N_{G_{i}} \frac{N_{G_{i}}}{N_{G_{i} \cap \emptyset_{j}}}\left(1+\frac{\kappa^{2}}{N_{O_{j}}}\right)^{2}\left(\kappa^{2} \frac{N_{G_{i}}}{N_{O_{j}}}\right)^{\left\lfloor\left(\frac{N_{G_{i}}}{N_{O_{j}}} \kappa^{2}-1\right)\right\rfloor .} .
\end{aligned}
$$

The constant $\kappa$ is small and is typically equal to $0.2 \frac{P}{D}$, where $P$ is the total perimeter of the object and $D$ is the image dimension. If $\frac{N_{G_{i}}}{N_{O_{j}}} \leq 50 \frac{D^{2}}{P^{2}}$, then the search is essentially quartic. In the worst case, $P \approx D$ and the requirement is $N_{G_{i}} \leq 50 N_{O_{j}}$, a very liberal bound. The term in the above equation that depends on the quality of the group, is the ratio $\frac{N_{G_{i}}}{N_{G_{i} \cap O_{j}}}$. This constitutes the first performance measure.

$$
P_{\text {time }}\left(G_{i}, O_{j}\right)=\frac{N_{G_{i} \cap O_{j}}}{N_{G_{i}}} .
$$

This measure ranges from zero to one and should be as large as possible to minimize the amount of search. The quality of the terminated constrained search will be proportional to the threshold, $t$, which is the number of model features explained by the group. Thus, the model to group match quality can be captured by $\frac{t}{N_{O_{j}}}$. Using this expression, coupled with the fact that the termination threshold $t$ is less than the number of common features between the model and the group, $N_{G_{i} \cap O_{j}}$, Borra and Sarkar [4] suggest the following as the second performance measure:

$$
P_{q u a l}\left(G_{i}, O_{j}\right)=\frac{N_{G_{i} \cap O_{j}}}{N_{O_{j}}} .
$$

The measure ranges from zero to one and should be large enough to ensure high-confidence recognition. High values of this measure will help discriminate between models, which will boost the recognition accuracy.

Consider the scenario that the grouping algorithm generates $N$ groups, $G_{1}, \ldots, G_{N}$ for an image with $K$ objects, $O_{1}, \ldots, O_{K}$. For each pair of the group, $G_{i}$ and the image object, $O_{j}$, that overlap, we compute $P_{\text {time }}\left(G_{i}, O_{j}\right)$ and $P_{\text {qual }}\left(G_{i}, O_{j}\right)$. Also, let $N_{\text {overlaps }}$ be the total number of overlaps, which can be anywhere between 0 and $N K$. We use the composite performance measure, $\beta$, formed by combining these measures as follows:

$$
\beta=\sqrt{\left(\frac{\sum_{i j} P_{\text {time }}\left(G_{i}, O_{j}\right)}{N_{\text {overlaps }}}\right)\left(\frac{\sum_{i j} P_{\text {qual }}\left(G_{i}, O_{j}\right)}{N_{\text {overlaps }}}\right)} .
$$

This product form of combination of the two individual measures tends to assign equal importance to the time and quality of recognition. In addition, the used normalized summation form for each of the measures tends to 1) penalize a group that is spread across two objects more than a group that overlaps with one object and the background, 2) prefer large groups over small groups, and 3) penalize groups of features that do not belong to any object.

Another possibility for performance measure are the false negative and false positive rates as measured by Amir 
and Lindenbaum [1], which are not directly tied to object recognition. We chose the option for task-based evaluation.

Since we are concerned with edge-based recognition, the features of interest are edge pixels. The manual construction of $3 \mathrm{D}$ models is cumbersome and not, to say the least, time consuming, and this renders the performance analysis almost intractable for real domains. We circumvent this problem by using manual outlines of object features in each image. Given an edge image, the collection of edge pixels close to the manual outline represents the perfect grouping of features in an image. In the case of 2D model-based recognition, particularly those that are view-based, the number of edge points will provide a good estimate of the number of model features.

\subsection{Image Set}

We use a database of 50 images. There are 10 images of natural objects in indoor backgrounds, 10 images of natural objects in outdoor backgrounds, 10 images of man-made objects in outdoor backgrounds, 10 images of man-made objects in indoor backgrounds, and 10 aerial images that contained man-made objects in natural surroundings. So, essentially, we have five different image classes. Each of these images is associated with manual ground truth outlines of the objects of interest. It took us approximately 30-60 minutes per image, depending on their size and also the complexity of the objects in the image, to ground truth each image. We avoided outlining ambiguous nonsharp edges, e.g., due to wrinkles on a shirt or statistical texture, e.g., texture on wood, but outline structural textures such as stripes of a zebra.

\subsection{Parameter Selection}

One of the important aspects of empirical performance evaluation is the choice of the parameters of the algorithm. The present grouping framework has 21 parameters: 13 are used in the Bayesian networks that are used to quantify the graph links, two parameters are used by the graph spectral partitioning (minimum cluster size and maximum partition strength), three are edge detection parameters, three parameters are used by the constant curvature contour segmentation algorithm.

We adopt a two-fold testing strategy for selecting the parameter combinations. As described earlier, we have five image classes comprising of 10 images each. Let us denote this by $C_{i}$, where $i \in\{1,2, \ldots, 5\}$. We divide each image class into two equal sets and denote this by $S_{1}^{i}$ and $S_{2}^{i}$, where $i$ indicates the image class. We select parameters based on training on $S_{1}^{i}$ (or $S_{2}^{i}$ ) and use them on $S_{2}^{i}$ (or $S_{1}^{i}$ ).

We train each cut on each image in the set. From this training, 10 best parameter combinations are extracted from each individual trained parameter set for each image and each cut measure. This gives us 30 trained parameter combinations for each image, 10 for each of the three cut measures, with a total of 150 parameter combinations for each image set, $S_{1}^{i}$ (or $S_{2}^{i}$ ). These 150 parameter sets are then applied to the images in the other corresponding set, i.e., parameter combinations trained on $S_{1}^{i}$ are applied to images in $S_{2}^{i}$ and vice versa. Thus, for each image and each cut we have 150 performance indices. Note that we trained parameters on a per image class basis and not on the whole (50) image set. In our earlier work [28], we found that optimal parameter for grouping was a function of image class. Thus, parameters that resulted in good performance, for say, aerial images, will not give good performance for indoor images.

In our analysis, we will consider the best, mean and range of the top 10 performances with these 150 performances on each image for each cut. We select these top 10 performances for each of the three cuts with the constraint that the same parameter combination sets are chosen. We rank the performances for the 150 parameter combinations for each cut measure and pick the parameter combinations that results the 10 lowest summed ranks over the three measures. While choosing these parameter combinations we pay attention to fix across cut measures only the parameters that determine the graph structure, i.e., the edge detection, constant curvature segmentation, and graph quantification parameters. The graph partitioning parameters are allowed to vary. This ensures that when we compare performances among the cut measures, they are on the same graph structure.

\subsection{Exploring the Parameter Space}

We select the optimal parameters by stochastically sampling the parameter space, which is effected by a team of learning automata [28], [7]. We associate one learning automaton with one algorithm parameter. A learning automaton (LA) is an algorithm that adaptively chooses from a set of possible actions on a random environment (the grouping algorithm and the images) so as to maximize the expected feedback (quality of the groups). Each automation uses a probability vector defined over the set of actions, $P\left(v_{1}\right), P\left(v_{2}\right), \cdots, P\left(v_{r}\right)$ to randomly choose between $r$ possible actions, $v_{1}, \cdots, v_{r}$. At the start, $P\left(v_{1}\right)=P\left(v_{2}\right)=$ $\cdots P\left(v_{r}\right)=1 / r$ so that each action is equally likely. The environment, which in our case includes the grouping algorithm and the image(s), evaluates the performance with the set of parameters, each individually chosen by an automaton. At each iteration, based on the feedback from the environment and past action history, the action probability vector is updated so that the action with large feedback is more likely to be chosen than those that resulted in small feedback values. Over time, the action that maximizes the feedback will be chosen.

It can be shown [20] that the team of learning automata is $\epsilon$-optimal, i.e., the team will converge close to global optimal values. The rate of convergence is dependent on the underlying learning rate, which we set to 0.1 . The traces of the average feedback from the environment increases with iterations. The usual recommended stopping condition is expressed in term of the probability of the optimal action being 


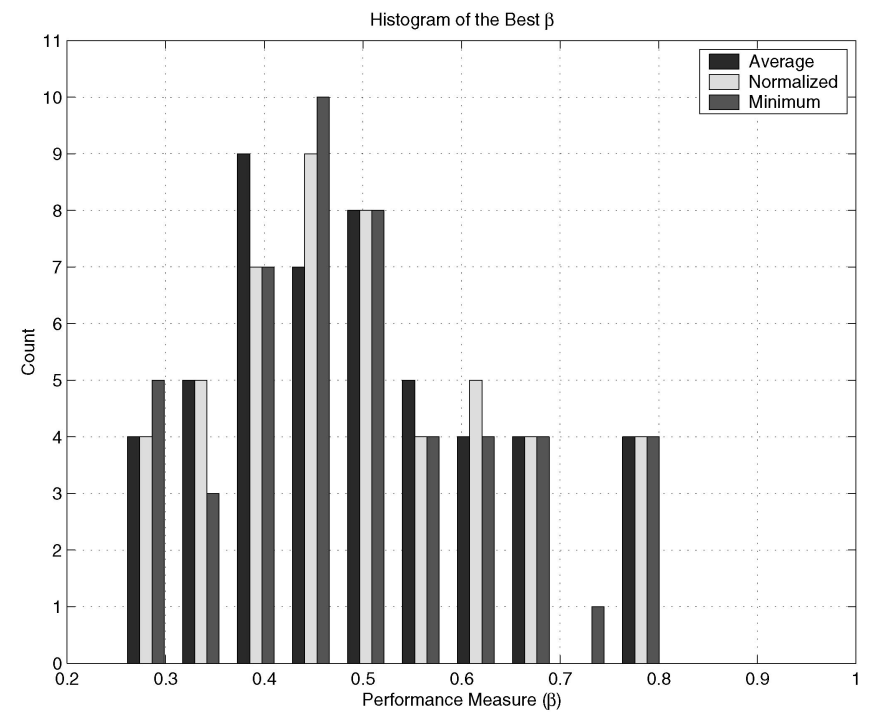

Fig. 5. Histogram of the best $\beta$ s (performance measure) for each of the cuts on all the 50 images.

high. However, since we are concerned just with selecting good parameter combinations, we decide to stop when the automata team is unable to find any parameter combination that is better than the previous ones for 100 consecutive iterations.

\subsection{Statistical Analysis}

We present detailed evaluation of the grouping performances with the three graph partitioning measures. In the following sections, we first consider the following questions: Are the best performances for each cut type significantly different? Is the variation of performance for each cut type significantly different from others? Is performance dependent on image class? We follow this by presentation of results for visual assessment and a discussion of time issues.

\subsubsection{Are the Best Performances for Each Cut Type Significantly Different?}

Does the performance for each of the partition cut measures significantly differ from the others for the best performance obtained from these parameter sets? Fig. 5 shows the histogram of the best performances, $\beta$ on each of the images. We see that the histograms overlap and we cannot clearly separate out one from the other. This gross observation can be refined by analysis of variances (ANOVA), ${ }^{3}$ which takes into account variations due to independent factors, which in this case are two-the image and the cut type. ANOVA unravels the impact of each source of variation on the total variation, which is listed in the first column of Table 1. The second column (DF) refers to the degrees of freedom (since there are three different cuts, the $D F$ for that factor is 2). The SS column lists the

3. Analysis of variance is a general statistical method for analyzing experimental data. Many texts on statistics describe analysis of variance methods. For example, see [24] for an introduction to the subject.
TABLE 1

ANOVA of the Best Performance on All Images

\begin{tabular}{|c|c|c|c|c|c|}
\hline Source & DF & SS & F-value & P-value & Comments \\
\hline Cut & 2 & 0.0006 & 0.69 & 0.5022 & Not Significant \\
\hline Image & 49 & 2.8558 & 122.37 & 0.0001 & Significant \\
\hline
\end{tabular}

Type I Sum of Squares. The F-value for each source is the ratio of the variance between different source values to the variance within the same source values. Larger F-values imply larger impact that the respective source factors have on the overall variation. Finally, the P-value tells us the level of significance of $H_{0}$, the NULL hypothesis viz, the variation due to the source is due to noise. If P-value is less than 0.05 , then with more than 95 percent confidence level can we reject the NULL hypothesis. From Table 1, we see that the cut type is not a significant source of variation (P-value is greater than 0.05). The major source of variation is the image set. So, it is clear that the best performances do not depend on the three cut measures used for grouping.

One of the criticisms against ANOVA is that it assumes normally distributed errors. So, we also considered nonparametric tests of the data. Friedman's test is chosen as this is a natural alternative for the repeated measures ANOVA test. The Friedman's test compares three or more paired groups. The data is first arranged in a tabular form where the rows are the possible combination of the values of the Cut or the Image factors. The columns list the performances $(\beta \mathrm{s})$. It then sorts the values in each row in ascending order and then sums the ranks in each column. If the column sums are significantly different, it will suggest that the effect of the factor is significant, else the observed variation is due to random errors.

Table 2 shows the results of five best performances applied on the cut measures $(\mathrm{Cut})$, with the performance measure $(\beta)$ as the dependent variable. Table 3 shows the results of five best performances applied on the images (Image), with the performance measure $(\beta)$ as the dependent variable. The first column of the table is the statistic considered. The second column shows the alternative hypothesis. The third column shows the degrees of freedom. The fourth column shows the value. The fifth column shows

TABLE 2

Friedman's Test on the Best Performances, Applied on Cut, with $\beta$ as the Dependent Variable

\begin{tabular}{|c|c|c|c|c|}
\hline \multicolumn{5}{|c|}{ Summary Statistics for Cut by $\beta$} \\
\hline \multicolumn{5}{|c|}{ Cochran-Mantel-Haenszel Statistics (Based on Rank Scores) } \\
\hline \hline Statistic & Alternative Hypothesis & DF & Value & P-value \\
\hline 1 & Nonzero Correlation & 1 & 0.045 & 0.8314 \\
\hline 2 & Row Mean Scores Differ & 2 & 0.047 & 0.9766 \\
\hline
\end{tabular}


TABLE 3

Friedman's Test on the Best Performances, Applied on Image, with $\beta$ as the Dependent Variable

\begin{tabular}{|c|c|c|c|c|}
\hline \multicolumn{5}{|c|}{ Summary Statistics for Image by $\beta$} \\
\hline \multicolumn{5}{|c|}{ Cochran-Mantel-Haenszel Statistics (Based on Rank Scores) } \\
\hline \hline Statistic & Alternative Hypothesis & DF & Value & P-value \\
\hline 1 & Nonzero Correlation & 1 & 30.228 & 0.0001 \\
\hline 2 & Row Mean Scores Differ & 49 & 145.903 & 0.0001 \\
\hline
\end{tabular}

the P-value. Observe that the row mean scores differ with a value of 0.047 and a P-value of 0.9766 , which implies that there is no statistical significance. In Table 3, we see the Pvalue associated with the row mean scores differ is 0.0001 , which means that images are a significant source of variation on the performance measure. We can gather the fact from observing both these tables that the cut measure is not significant and the images are significant when the $\beta$ s are considered on rank scores.

\subsubsection{Is the Variation of Performance for Each Cut Type Significantly Different from Each Other?}

We have already shown that the cut type does not make a significant difference on the best performance over all the image sets. Here, we consider the variation in performance, which we quantify by the difference between the best performance and the tenth best performance on each image, $\beta_{1-10}=\beta_{1}-\beta_{10}$. Table 4 lists the ANOVA results. We can see that the range of performance $\beta_{1-10}$ for the cut types are not significantly different.
TABLE 4

ANOVA of the Range of the 10 Best Performance $\beta_{1-10}=\beta_{1}-\beta_{10}$ on All Images

\begin{tabular}{|c|c|c|c|c|c|}
\hline Source & DF & SS & F-value & P-value & Comments \\
\hline Cut & 2 & 0.0033 & 1.36 & 0.2617 & Not Significant \\
\hline Image & 49 & 0.6356 & 10.43 & 0.0001 & Significant \\
\hline
\end{tabular}

\subsubsection{Is Performance Dependent on Image Class?}

As we have described above, we have five classes of images in the data set. The question we ask is: Is the performance of the grouping-by-partitioning strategy, irrespective of the partitioning measure used, dependent on the image class type? Table 5 lists the mean values of the best performances, along with 95 percent confidence intervals, for each image class and for each cut type. We observe clearly that, in each image class, there is not much variation in performance with respect to the cut measure. We also observe that, in particular, the performances for images of man made objects in indoor surroundings and aerial images are lower than for other image classes.

To establish the statistical significances of our observation, we conducted ANOVA. Here, we consider the best parameter combination for each image selected as discussed in Section 4.5. The two independent factors, the cut measure and the image class, along with their interaction are the possible sources of variations. The results of ANOVA are shown in Table 6, where we see that, the variation of performance due to the cut measure is not significant. In addition, the interaction between the image class and the cut type is also not significant. Thus, it is not likely the case that

TABLE 5

Mean Values with 95 Percent Confidence Level of the Best Performance Index, $\beta$, for Each Class of Images Considered for Each Cut Type

\begin{tabular}{|c|c|c|c|c|}
\hline Image Class & Cut & Lower CL & Upper CL & Mean \\
\hline \multirow{3}{*}{$\begin{array}{c}1 \\
\text { (Natural objects with indoor environment) }\end{array}$} & Average & 0.49 & 0.52 & 0.51 \\
\hline & Normalized & 0.50 & 0.53 & 0.52 \\
\hline & Minimum & 0.51 & 0.54 & 0.52 \\
\hline \multirow{3}{*}{$\begin{array}{c}2 \\
\text { (Natural objects with outdoor environment) }\end{array}$} & Average & 0.46 & 0.52 & 0.49 \\
\hline & Normalized & 0.49 & 0.55 & 0.52 \\
\hline & Minimum & 0.49 & 0.56 & 0.53 \\
\hline \multirow{3}{*}{$\begin{array}{c}3 \\
\text { (Man-made objects with indoor environment) }\end{array}$} & Average & 0.36 & 0.40 & 0.38 \\
\hline & Normalized & 0.36 & 0.40 & 0.38 \\
\hline & Minimum & 0.37 & 0.40 & 0.39 \\
\hline \multirow{3}{*}{$\begin{array}{c}4 \\
\text { (Man-made objects with outdoor environment) }\end{array}$} & Average & 0.42 & 0.49 & 0.45 \\
\hline & Normalized & 0.45 & 0.52 & 0.49 \\
\hline & Minimum & 0.46 & 0.53 & 0.50 \\
\hline \multirow{3}{*}{$\begin{array}{c}5 \\
\text { (Aerial) }\end{array}$} & Average & 0.34 & 0.36 & 0.35 \\
\hline & Normalized & 0.34 & 0.36 & 0.35 \\
\hline & Minimum & 0.32 & 0.35 & 0.34 \\
\hline
\end{tabular}


TABLE 6

ANOVA of the Best Performance on All Images with Interactions between the Image Class and the Cut Type

\begin{tabular}{|c|c|c|c|c|c|}
\hline Source & DF & SS & F-value & P-value & Comments \\
\hline Cut & 2 & 0.0006 & 0.02 & 0.9804 & Not Significant \\
\hline Image Class & 4 & 0.6444 & 9.65 & 0.0001 & Significant \\
\hline Image Class*Cut & 8 & 0.0046 & 0.03 & 1.0000 & Not significant \\
\hline
\end{tabular}

one cut measure is appropriate for one image class while another cut measure performs better for another image class. However, the variation due to the image classes is significant. Thus, the performance variations between image classes that we see in Table 5 are significant. The performances for images of man-made objects in indoor surroundings and aerial images are lower than for other image classes.

\subsubsection{Visual Assessment of Results}

In this section, we present final grouping results on some images for visual assessment, which because of its subjective nature has to be done with caution. Visual assessment might not agree with the computed performance measure, $\beta$. In particular, we have to keep in mind that our performance measure 1) penalizes groups that straddles two objects more than groups that include part of an object and the background and 2) penalizes small groups.

Fig. 6 shows three images on which all cut measures performs equally well as per visual assessment. Note that the parameter set was the same for all the three cuts for each image. Thus, the three cut strategies operated on the same graph. Figs. 6b, 6c, and $6 \mathrm{~d}$ shows the best performances of all the cuts. In Figs. 6f, 6g, and 6h, we see the vehicle clearly segmented out. Observe that the clutter due to the trees and the buildings are thrown out. All the cut algorithms are able to form correct grouping. In Figs. 6j, 6k, and 6l, we see the performances of all the cuts on a zebra image. Clearly, all the cut algorithms are able to separate out the regularly stripped zebra from the grass background. On our Web site (http://marathon.csee.usf.edu/PO/Cuts.html), we show the results on all the 50 images, for each cut type.

Fig. 7 shows the performances on some images on which the cut measures differ the most. In Fig. 7a, the average cut performs the best. Note that most of the background clutter is removed and the chair is grouped properly. The normalized cut is best for Fig. 7e and the min-cut is the best in Fig. 7i. However, as we have seen earlier, this dependence of performance of each cut type on the image is not statistically significant. Variation due to the image type swamps any variation due to the cut type. This reinforces the argument against the reliance on just visual assessment of results on a few images.

\subsubsection{Time Issues}

Table 7 shows the basic statistics of the time in seconds, taken by each cut algorithms to finish the grouping process, including low-level processing and graph construction, which are the same for all the cut measures. The algorithms were run on a Sun Ultra-Enterprise with a clock speed of $247 \mathrm{MHz}$. We can clearly see that the time taken to compute the normalized cut vary more than the other two cuts. This can be attributed to the normalized Laplacian matrix structure, all of whose diagonal entries are one and the rest of the entries are negative but with absolute values less than one. Whereas for the average cut, the diagonal elements can have a value greater than 1 . Hence, the relative variation between the matrix element is less for the normalized cut case, which effects the rate of convergence of the eigenvalue/eigenvector computations. Effect of the complexities of computing the eigenvectors of the (normalized and unnormalized) Laplacian matrix can probably be reduced using well-known linear algebra tricks and approximations, which we did not explore in this study.

\section{Conclusions, Implications, ANd Remarks}

We theoretically analyzed the form of three grouping measures, the min-cut, average-cut, and normalized-cut, using random weighted graph models and the concept of stochastic order. The focus of the analyses was to understand for what kinds of image statistics would optimizing a measure, irrespective of the particular algorithm used, result in correct partitioning. Another question of interest was if the recursive bipartitioning strategy can separate out groups corresponding to $K$ objects from each other. We find that none of the measures seem to be appropriate for all image statistics. The conclusions are that under very restrictive conditions when the average interobject feature affinity is very weak when compared to the average intraobject feature affinity, the minimum cut measure is optimal. The average cut measure is optimal for graphs whose partition width is less than the mode of distribution of all possible partition widths. The normalized cut measure is optimal for a more restrictive subclass of graphs whose partition width is less than the mode of the partition width distributions and the strength of interobject links is six times less than the intraobject links. Since any theoretical analysis is limited by the necessary use of many implicit and explicit assumptions, we also studied empirically, whether the cut measures, despite their theoretical limitations, actually matter in practice. We considered the context of grouping extended edge segments for object recognition using constrained search. We find that the performances of the three cut measures are statistically equivalent. However, the variation due to the image classes is significant; the performance with the three measures is worst for manmade objects in indoor surroundings and for aerial images. 


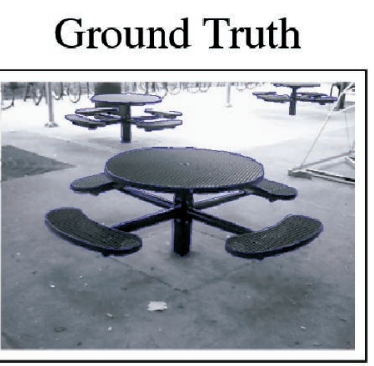

(a)

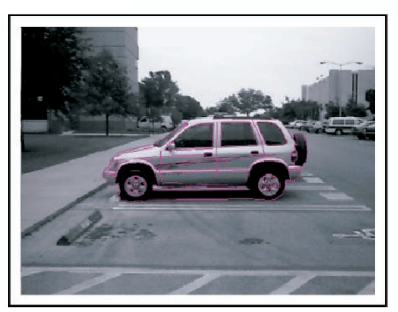

(e)

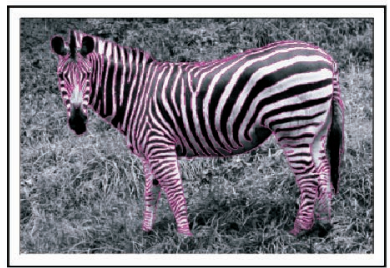

(i)

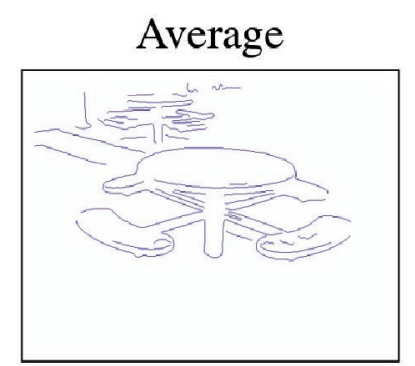

(b)

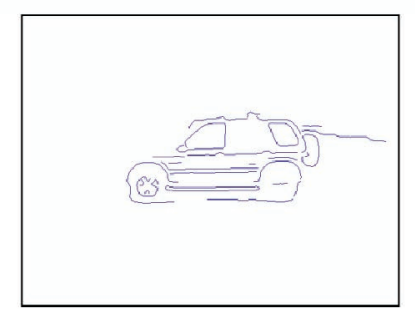

(f)

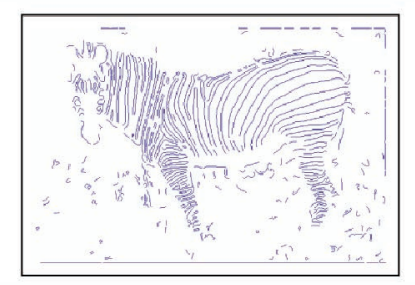

(j)

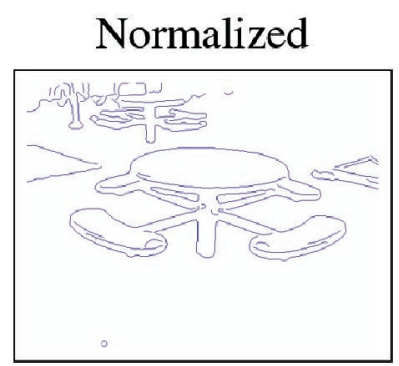

(c)

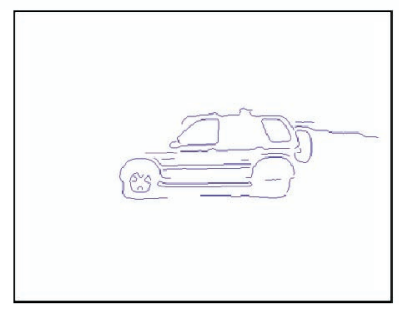

(g)

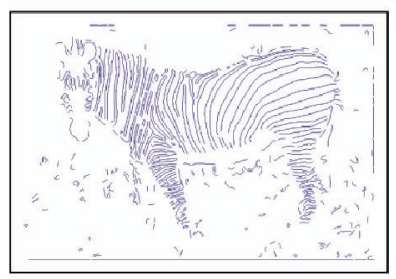

$(\mathrm{k})$

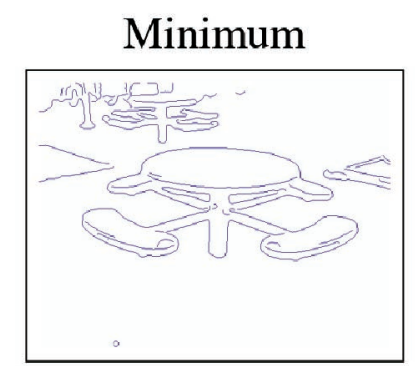

(d)

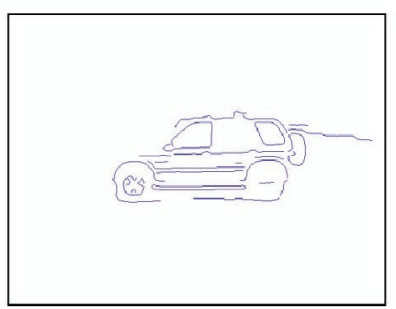

(h)

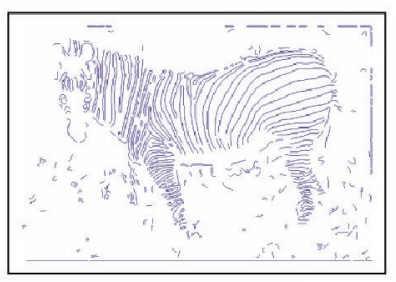

(l)

Fig. 6. (b), (c), and (d) $\beta=0.79$. (f), (g), and (h) $\beta=0.75$. (j), (k), and (I) $\beta=0.80$. Images in which all three cuts perform equally well. The first column shows the original image along with the manually drawn ground truth, the second column shows the performance for the average Cut, the third column for the normalized cut, and the fourth column for the min-cut.

Interestingly, the interaction between the image class and the cut type is also not significant.

So, what are the implications of the conclusions that we have drawn from this study? First, the choice of the nature of the cut measures, while theoretically important, does not seem to be critically important from an empirical standpoint. The average cut measure seems to be somewhat a better choice from a theoretical optimality point of view and time considerations. Second, the importance of learning grouping parameters on a per image basis is obvious. Performances can vary greatly with the chosen parameters. Third, image type dominate as a source of variation in performance. Poor performance on certain image types such as aerial and man-made objects in indoor surroundings can be attributed to the fact that the statistics of the objects of interest and the background are similar. Relations that are richer than just 2-ary ones need to be considered when constructing the scene structure (hyper)graph. Just 2-ary relations are probably inadequate for these domains.

The analysis in this paper is by no means the final word on the topic of the appropriateness of the various cut measures. The empirical study can be extended to include other types of low-level features, such as image pixels, with different forms of the affinity function. The larger the scope of the variations studied, the more reliable would be the conclusions, however, empirical studies can never establish conclusions with absolute certainty. This is why we augmented it with theoretical analysis of the measures. There are various directions along which the theoretical analysis can be extended, some of which are probably mathematically intractable. First, the independence assumption between the links of the scene structure can be replaced with at least a local dependence assumption. Second, presently there is an implicit assumption that the cut values for the various partition classes are independent of each other, which is not true. However, we do expect the dependence to reduce sharply with the addition of additional independent components as the difference between the partition classes increase. Third, it would be nice if we could make the following stochastic comparison $\min _{\mathbf{f} \in \Delta} \Sigma(\mathbf{f})<\min _{\mathbf{f} \notin \Delta} \Sigma(\mathbf{f})$. We believe that the present analysis is on the conservative side and this comparison of the minimum would give a truer picture. But, this would require the derivation of the distribution function (quite possibly related to the Weibull distribution) of the minimum of nonidentically distributed random variables. A possible starting point would be the body of work in extreme valued statistics [11], but designed for non-iid variables. Last, of course, is the open question: In the stochastic order sense, what is the form of the optimal cut measure? If there is one! 


\section{Ground Truth}

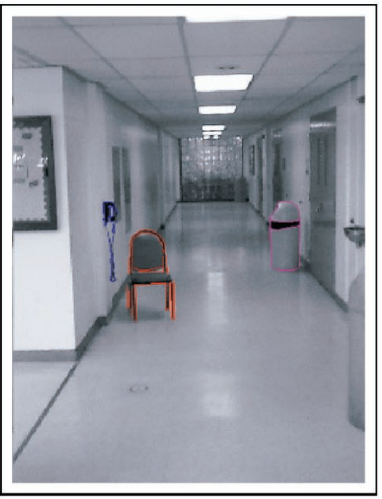

(a)

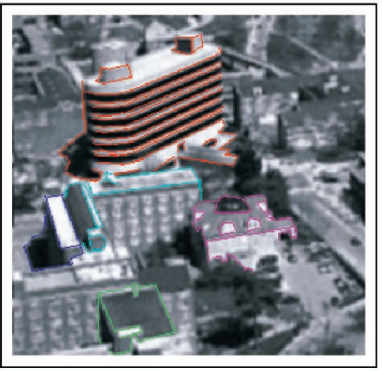

(e)

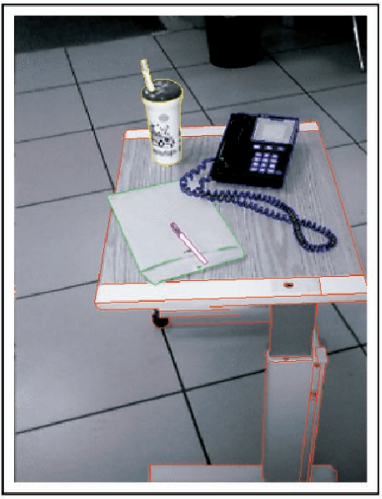

(i)

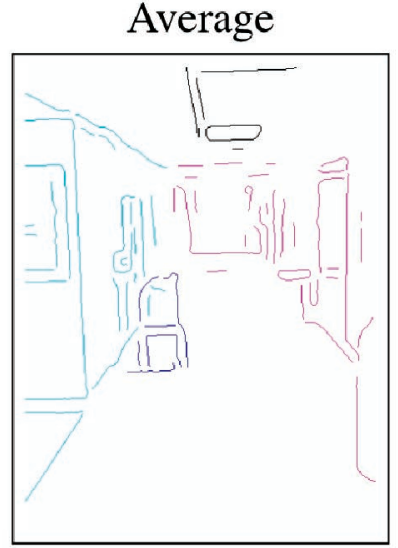

(b)

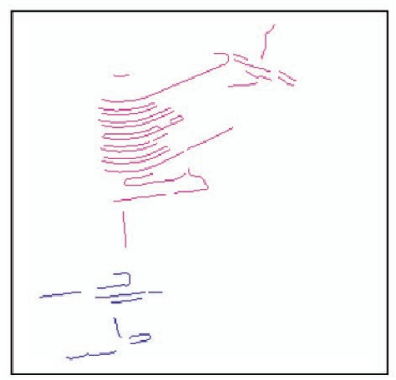

(f)

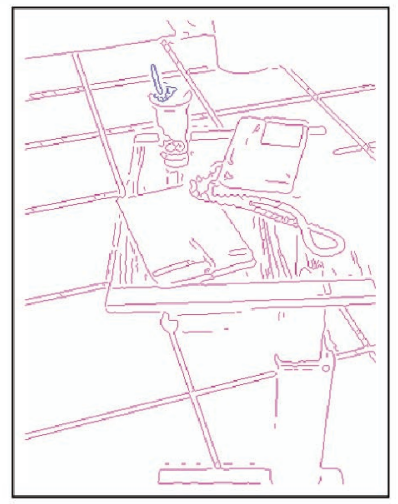

(j)

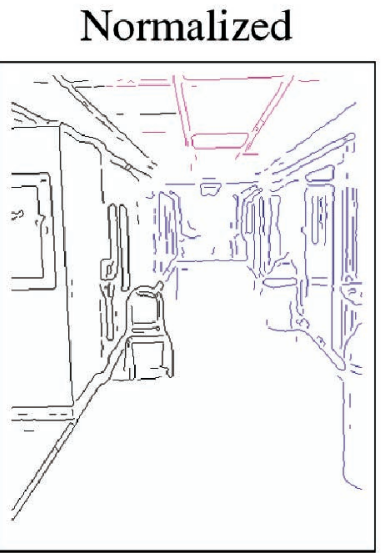

(c)

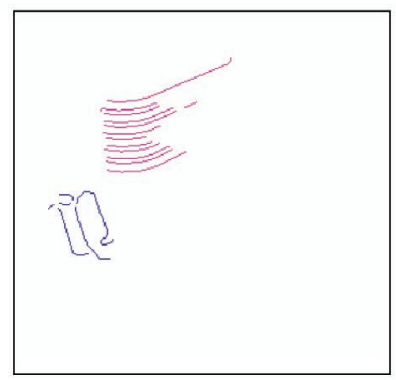

(g)

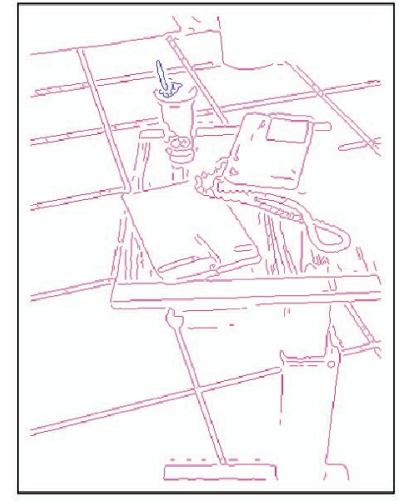

(k)

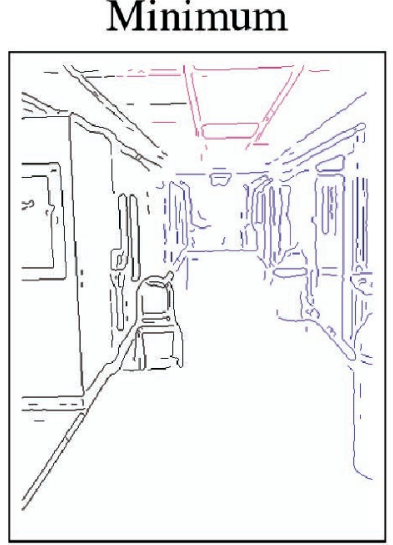

(d)

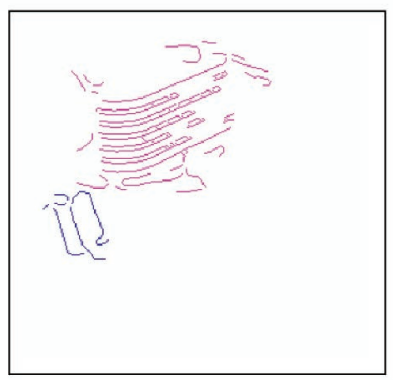

(h)

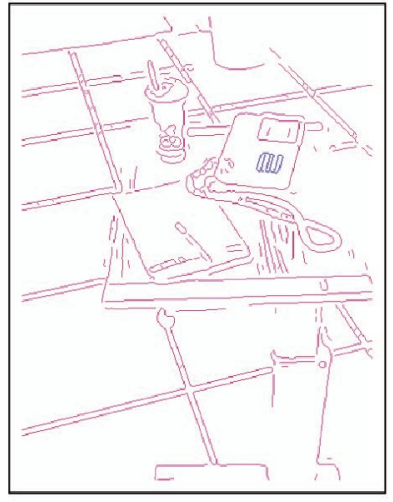

(I)

Fig. 7. (b) $\beta=0.35$. (c) $\beta=0.25$. (d) $\beta=0.25$. (f) $\beta=0.35$. (g) $\beta=0.40$. (h) $\beta=0.38$. (j) $\beta=0.35$. (k) $\beta=0.35$. (l) $\beta=0.43$. Sample of images on which the performance of one cut is the most different from the other.

\section{ACKNOWLEDGMENTS}

This work was supported by US National Science Foundation CAREER grant nos. IIS-9907141 and EIA-0130768.

TABLE 7

Min, Max, Mean, and Median of the Time Taken by the Average, Normalized, and Minimum Cut

\begin{tabular}{|c|r|r|r|}
\hline & Average & Normalized & Minimum \\
\hline Min & 1.06 & 1.15 & 1.97 \\
\hline Max & 81.54 & 355.91 & 99.58 \\
\hline Mean & 7.76 & 27.11 & 17.27 \\
\hline Median & 6.15 & 9.02 & 16.4 \\
\hline
\end{tabular}

\section{REFERENCES}

[1] A. Amir and M. Lindenbaum, "A Generic Grouping Algorithm and Its Quantitative Analysis," IEEE Trans. Pattern Analysis and Machine Intelligence, vol. 20, no. 2, pp. 168-185, Feb. 1998.

[2] A. Berengolts and M. Lindenbaum, "On the Performance of Connected Components Grouping," Int'l J. Computer Vision, vol. 41, no. 3, pp. 195-216, 2001.

[3] R.P. Boppana, "Eigenvalues and Graph Bisection: An Average Case Analysis," Proc. 28th Int'l Symp. Foundations of Computer Science, pp 280-285, 1987.

[4] S. Borra and S. Sarkar, "A Framework for Performance Characterization of Intermediate-Level Grouping Modules," IEEE Trans. Pattern Analysis and Machine Intelligence, vol. 19, no. 11, pp. 1306-1312, Nov. 1997.

[5] T. Bui, S. Chaudhuri, T. Leighton, and M. Sipser, "Graph Bisection Algorithms with Good Average Case Behavior," Proc. 25th Int'l Symp. Foundations of Computer Science, pp. 181192, 1984. 
[6] S. Casadei and S.K. Mitter, "Hierarchical Image SegmentationDetection of Regular Curves in a Vector Graph," Int'l J. Computer Vision, vol. 27, no. 1, pp. 71-100, 1998.

[7] S. Chavali and S. Sarkar, "Modeling Parameter Space Behavior of Vision Systems Using Bayesian Networks," Computer Vision and Image Understanding, vol. 79, pp. 185-223, 2000.

[8] D.T. Clemens and D.W. Jacobs, "Space and Time Bounds on Indexing 3D Models from 2D Images," IEEE Trans. Pattern Analysis and Machine Intelligence, vol. 13, no. 10, pp. 1007-1017, Oct. 1991.

[9] J. Costeira and T. Kanade, "A Multibody Factorization Method for Motion Analysis," Proc. Int'l Conf. Computer Vision, pp. 1071-1076, 1995.

[10] I.J. Cox and S.B.R. an, and Y. Zhong, “Ratio Regions: A Technique for Image Segmentation," Proc. Int'l Conf. Pattern Recognition, pp. 557-564, 1996.

[11] J. Galambos, The Asymptotic Theory of Extreme Order Statistics. John Wiley and Sons, 1978.

[12] Y. Gdalyahu, N. Shental, and D. Weinshall, "Perceptual Grouping and Segmentation by Stochaistic Clustering," Proc. IEEE CS Conf. Computer Vision and Pattern Recognition, vol. 1, pp. 367-374, June 2000.

[13] W.E.L. Grimson, "The Combinatorics of Heuristic Search Termination for Object Recognition in Cluttered Environments," IEEE Trans. Pattern Analysis and Machine Intelligence, vol. 13, no. 9, pp. 920-935, Sept. 1991

[14] G. Guy and G. Medioni, “Inferring Global Perceptual Contours from Local Features," Int'l J. Computer Vision, vol. 20, pp. 113-133, 1996.

[15] L. Herault and R. Horaud, "Figure Ground Discrimination: A Combinatorial Optimization Method," IEEE Trans. Pattern Analysis and Machine Intelligence, vol. 15, no. 9, pp. 899-914, Sept. 1993.

[16] D.P. Huttenlocher and P.C. Wayner, "Finding Convex Edge Groupings in an Image," Proc. Int'l Conf. Computer Vision and Pattern Recognition, pp. 406-412, 1991.

[17] D.W. Jacobs, "Robust and Efficient Detection of Salient Convex Groups," IEEE Trans. Pattern Analysis and Machine Intelligence, vol. 18, no. 1, pp. 23-37, Jan. 1996.

[18] I.H. Jermyn and H. Ishikawa, "Globally Optimal Regions and Boundaries as Minimum Ratio Weight Cycles," IEEE Trans. Pattern Analysis and Machine Intelligence, vol. 23, no. 10, pp. 10751088, Oct. 2001

[19] D.W. Matula, "Graph Theoretic Techniques for Cluster Analysis Algorithms," Classification and Clustering, J.V. Ryzin, ed., pp. 95-129, 1977.

[20] K.S. Narendra and M.L. Thathachar, Learning Automata: An Introduction. Prentice Hall, 1989.

[21] P. Perona and W. Freeman, "A Factorization Approach to Grouping," Proc. European Conf. Computer Vision, pp. 655-670, 1998.

[22] S.V. Raman, S. Sarkar, and K.L. Boyer, "A Graph-Theoretic Approach to Generating Structure Hypotheses in Cerebral Magnetic Resonance Images," Computer Vision, Graphics, and Image Understanding, vol. 57, no. 1, pp. 81-98, Jan. 1993.

[23] Y. Saab and V. Rao, "On the Graph Bisection Problem," IEEE Trans. Circuits and Systems, vol. 39, no. 9, pp. 760-762, Sept. 1992.

[24] T. Sanocki, Student Friendly Statistics. Prentice Hall, 2000.

[25] H. Saran and V.V. Vazirani, "Finding K-Cuts within Twice the Optimal," SIAM J. Computing, vol. 24, no. 1, pp. 101-108, Feb. 1995.

[26] S. Sarkar and K.L. Boyer, "A Computational Structure for Preattentive Perceptual Organization: Graphical Enumeration and Voting Methods," IEEE Trans. Systems, Man, and Cybernetics, vol. 24, no. 2, pp. 246-267, Feb. 1994.

[27] S. Sarkar and K.L. Boyer, "Quantitative Measure of Change Based on Feature Organization: Eigenvalues and Eigenvectors," Computer Vision and Image Understanding, vol. 71, no. 1, pp. 110-136, July 1998.

[28] S. Sarkar and P. Soundararajan, "Supervised Learning of Large Perceptual Organization: Graph Spectral Partitioning and Learning Automata," IEEE Trans. Pattern Analysis and Machine Intelligence, vol. 22, no. 5, pp. 504-525, May 2000.

[29] G.L. Scott and H.C. Longuet-Higgins, "Feature Grouping by Relocalisation of Eigenvectors of the Proximity Matrix," Proc. British Machine Vision Conf., pp. 103-108, 1990.

[30] J. Sha'ashua and S. Ullman, "Structural Saliency: The Detection of Globally Salient Structures Using a Locally Connected Network," Proc. Int'l Conf. Computer Vision, pp. 321-327, 1988.

[31] M. Shaked and J.G. Shantikumar, Stochastic Orders and Their Application. Academic Press Inc., 1994.
[32] J. Shi and J. Malik, "Normalized Cuts and Image Segmentation," Proc. IEEE CS Conf. Computer Vision and Pattern Recognition, pp. 731-737, June 1997.

[33] P. Soundararajan and S. Sarkar, "Investigation of Measures for Grouping by Graph Partitioning," Proc. Int'l Conf. Computer Vision and Pattern Recognition, pp. 239-246, 2001.

[34] M. Stoer and F. Wagner, "A Simple Min Cut Algorithm," Algorithms-ESA '94, pp. 141-147, 1994.

[35] C.C. Tu and H. Cheng, "Spectral Methods for Graph Bisection Problems," Computers Operations Research, vol. 25, nos. 7/8, pp. 519-530, July 1999.

[36] D. Wagner and F. Wagner, "Between Min Cut and Graph Bisection," Technical Report no. 307/1991, Algorithmic Discrete Math. Report, TU Berlin, Sept. 1991.

[37] S. Wang and J.M. Siskind, "Image Segmentation with Minimum Mean Cut," Proc. Int'l Conf. Computer Vision, pp. 517-524, 2001.

[38] Y. Weiss, "Segmentation Using Eigenvectors: A Unifying View," Proc. Int'l Conf. Computer Vision, vol. 2, pp. 975-982, 1999.

[39] L.R. Williams and D.W. Jacobs, "Stochastic Completion Fields: A Neural Model of Illusory Contour Shape and Salience," Proc. Int'l Conf. Computer Vision, pp. 408-415, 1995.

[40] L.R. Williams and K.K. Thornber, "A Comparison of Measures for Detecting Natural Shapes in Cluttered Backgrounds," Int'l J. Computer Vision, vol. 34, nos. 2/3, pp. 81-96, Aug. 1999.

[41] Z. Wu and R. Leahy, "An Optimal Graph Theoretic Approach to Data Clustering: Theory and Application to Image Segmentation," IEEE Trans. Pattern Analysis and Machine Intelligence, vol. 15, no. 11, pp. 1101-1113, Nov. 1993.

[42] C.T. Zahn, "Graph Theoretic Methods for Detecting and Describing Gestalt Clusters," IEEE Trans. Computers, vol. 20, pp. 68-86, 1971.

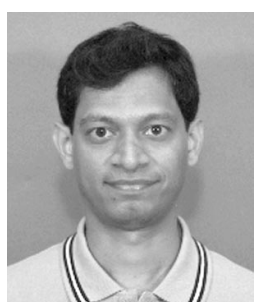

Padmanabhan Soundararajan received the $\mathrm{BE}$ degree in electronics and communication from Mysore University, India, in 1995. From 1995 to 1998, he was a project assistant at the Indian Institute of Science, Bangalore. He joined the Computer Science and Engineering Department at the University of South Florida in 1998 and is currently pursuing the $\mathrm{PhD}$ degree. His research interests include perceptual organization, statistical analysis for grouping, and performance evaluation of vision systems. He is a student member of IEEE and IEEE Computer Society.

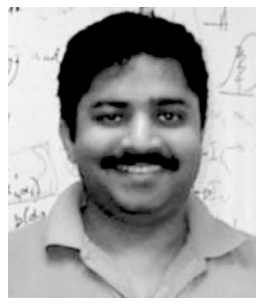

Sudeep Sarkar received the BTech degree in electrical engineering from the Indian Institute of Technology, Kanpur, in 1988, where he was judged the best graduating electrical engineer. $\mathrm{He}$ received the MS and $\mathrm{PhD}$ degrees in electrical engineering, on a University Presidential Fellowship, from The Ohio State University, Columbus, in 1990 and 1993, respectively. Since 1993, he has been with the Computer Science and Engineering Department at the University of South Florida, Tampa, where he is currently an associate professor. He was the recipient of the US National Science Foundation CAREER award in 1994, the USF Teaching Incentive Program Award for undergraduate teaching excellence in 1997, and the Outstanding Undergraduate Teaching Award in 1998. He is the coauthor of the book Computing Perceptual Organization in Computer Vision, published by World Scientific. He is also the coeditor of the book Perceptual Organization for Artificial Vision Systems published by Kluwer Publishers. He was the guest coeditor of the Computer Vision and Image Understanding (CVIU) journal special issue on perceptual organization in computer vision, October 1999. He is presently serving on the editorial boards for the IEEE Transactions on Pattern Analysis and Machine Intelligence and the Journal of Pattern Recognition. He has served on the editorial board of Pattern Analysis Applications Journal during 20002001. His research interests include perceptual organization in single image and multiple image sequences, probabilistic reasoning, Bayesian Networks, low-level image segmentation, color-texture analysis of burn scars, nonrigid modeling of burn scars, and performance evaluation of vision systems. He is a member of the IEEE and the IEEE Computer Society. 